\title{
DIVISORS OF SHIFTED PRIMES
}

\author{
DIMITRIS KOUKOULOPOULOS
}

\begin{abstract}
We bound from below the number of shifted primes $p+s \leq x$ that have a divisor in a given interval $(y, z]$. Kevin Ford has obtained upper bounds of the expected order of magnitude on this quantity as well as lower bounds in a special case of the parameters $y$ and $z$. We supply here the corresponding lower bounds in a broad range of the parameters $y$ and $z$. As expected, these bounds depend heavily on our knowledge about primes in arithmetic progressions. As an application of these bounds, we determine the number of shifted primes that appear in a multiplication table up to multiplicative constants.
\end{abstract}

\section{IntRoduction}

When one studies the multiplicative structure of the integers a natural and important question that arises is how many integers possess a divisor in a prescribed interval $(y, z]$. More precisely, for $y<z$ and $x \geq 1$ define

$$
H(x, y, z)=\mid\{n \leq x: \exists d \mid n \text { with } y<d \leq z\} \mid .
$$

The study of this function was initiated by Besicovitch [2] and was further developed by Erdős [6], 7], 9] and Tenenbaum [24], 25], who obtained bounds on $H(x, y, z)$ in various cases of the parameters $y$ and $z$. In his seminal paper [26] Tenenbaum focused on estimating $H(x, y, z)$ for all $x, y, z$ and he obtained reasonably sharp bounds on it. A consequence of Tenenbaum's work was the realization that, for fixed $x$ and $y$, as $z$ varies in $(y, x]$ the behavior of $H(x, y, z)$ changes when $z$ is around $y+y(\log y)^{-\log 4+1}, 2 y$ and $y^{2}$. The problem of establishing the correct order of magnitude of $H(x, y, z)$ was completely resolved by Ford in his profound work [11], where he discovered a striking connection between the distribution of the prime factors of integers with a divisor in $(y, z]$ and random walks with certain constraints. We state here the core of the main theorem in [11]. First, for a given pair $(y, z)$ with $2 \leq y<z$ define $\eta, u, \beta$ and $\xi$ by

$$
z=e^{\eta} y=y^{1+u}, \quad \eta=(\log y)^{-\beta}, \quad \beta=\log 4-1+\frac{\xi}{\sqrt{\log \log y}} .
$$

Furthermore, put

$$
\begin{aligned}
z_{0}(y) & =y \exp \left\{(\log y)^{-\log 4+1}\right\} \approx y+y(\log y)^{-\log 4+1}, \\
G(\beta) & = \begin{cases}\frac{1+\beta}{\log 2} \log \left(\frac{1+\beta}{e \log 2}\right)+1 & 0 \leq \beta \leq \log 4-1, \\
\beta & \log 4-1 \leq \beta,\end{cases}
\end{aligned}
$$

Date: November 20, 2018.

2000 Mathematics Subject Classification. Primary: 11N25, secondary: 11N13. 
and

$$
\delta=1-\frac{1+\log \log 2}{\log 2}=0.086071 \ldots
$$

Lastly, here and for the rest of this paper the notation $f \asymp g$ means that $f \ll g$ and $g \ll f$. Constants implied by $\ll$, $\gg$ and $\asymp$ are absolute unless otherwise specified, e.g. by a subscript.

Theorem 1.1 (Ford [11]). Let $x>100000$ and $100 \leq y \leq z-1$ with $z \leq x$.

(a) If $y \leq \sqrt{x}$, then

$$
\frac{H(x, y, z)}{x} \asymp \begin{cases}\log (z / y)=\eta & y+1 \leq z \leq z_{0}(y), \\ \frac{\beta}{\max \{1,-\xi\}(\log y)^{G(\beta)}} & z_{0}(y) \leq z \leq 2 y, \\ u^{\delta}\left(\log \frac{2}{u}\right)^{-3 / 2} & 2 y \leq z \leq y^{2} \\ 1 & z \geq y^{2} .\end{cases}
$$

(b) If $y>\sqrt{x}$, then

$$
H(x, y, z) \asymp \begin{cases}H\left(x, \frac{x}{z}, \frac{x}{y}\right) & \frac{x}{y} \geq \frac{x}{z}+1, \\ \eta x & \text { else. }\end{cases}
$$

When the interval $(y, z]$ is relatively short, Tenenbaum established an asymptotic formula for $H(x, y, z)$.

Theorem 1.2 (Tenenbaum [26]). If $z \leq \sqrt{x}$ and $\xi \rightarrow \infty$, then

$$
H(x, y, z) \sim \eta x \quad(y \rightarrow \infty, z-y \rightarrow \infty) .
$$

A natural generalization of $H(x, y, z)$ arises from restricting the range of $n$ to be some subset of the natural numbers $\mathscr{A}$. To this end we define

$$
H(x, y, z ; \mathscr{A})=\mid\{n \in[0, x] \cap \mathscr{A}: \exists d \mid n \text { with } y<d \leq z\} \mid .
$$

If $\mathscr{A}$ is reasonably well-distributed in arithmetic progressions, then a simple heuristic shows that we should have

$$
H(x, y, z ; \mathscr{A}) \approx \frac{|\mathscr{A} \cap[0, x]|}{x} H(x, y, z) .
$$

In the case that $\mathscr{A}$ is an arithmetic progression Ford, Khan, Shparlinski and Yankov [12] obtained upper bounds on $H(x, y, z ; \mathscr{A})$. In the present paper we focus on the special and important case when $\mathscr{A}=P_{s}:=\{p+s: p$ prime $\}$ for fixed $s \neq 0$. It is well-known that $P_{s}$ is well-distributed in arithmetic progressions $a(\bmod q)$ with $(a-s, q)=1$. Making this precise using sieving arguments and combining it with the methods developed in [11] can lead to bounds on $H\left(x, y, z ; P_{s}\right)$ of the expected order of magnitude. The upper bounds were settled by Ford in [11]. We state below a short interval version of Theorem 6 in [11]; for a proof of it see the proofs of Theorem 6 and Lemma 6.1 in [11]. 
Theorem 1.3 (Ford [11]). Fix $s \in \mathbb{Z} \backslash\{0\}$. Let $2 \leq y \leq \sqrt{x}, y+1 \leq z \leq x$ and $x(\log z)^{-10} \leq \Delta \leq x$. Then

$$
H\left(x, y, z ; P_{s}\right)-H\left(x-\Delta, y, z ; P_{s}\right) \ll_{s} \begin{cases}\frac{\Delta}{x} \frac{H(x, y, z)}{\log x} & z \geq y+(\log y)^{2 / 3}, \\ \frac{\Delta}{\log x} \sum_{\substack{y<d \leq z \\(d, s)=1}} \frac{1}{\phi(d)} & z \leq y+(\log y)^{2 / 3} .\end{cases}
$$

Remark 1.1. The reason that the upper bound in Theorem 1.3 has this particular shape is due to our incomplete knowledge about the sum $\sum_{y<d \leq z} \frac{1}{\phi(d)}$ when the interval $(y, z]$ is very short. The main theorem in [23] implies that

$$
\sum_{y<d \leq z} \frac{1}{\phi(d)} \asymp \log (z / y) \quad\left(z \geq y+(\log y)^{2 / 3}\right),
$$

whereas standard conjectures on Weyl sums would yield that

$$
\sum_{y<d \leq z} \frac{1}{\phi(d)} \asymp \log (z / y) \quad(z \geq y+\log \log y) .
$$

The range of $y$ and $z$ in (1.2) is the best possible one can hope for, since it is well-known that the order of $n / \phi(n)$ can be as large as $\log \log n$ if $n$ has many small prime factors.

In general, lower bounds on $H\left(x, y, z ; P_{s}\right)$ are more difficult because they rely on more precise knowledge about the distribution of primes in arithmetic progressions, which is a notoriously difficult problem. A special case was worked out by Ford.

Theorem 1.4 (Ford [11]). For fixed $s, a, b$ with $s \in \mathbb{Z} \backslash\{0\}$ and $0 \leq a<b \leq 1$ we have

$$
H\left(x, x^{a}, x^{b} ; P_{s}\right) \gg_{s, a, b} \frac{x}{\log x} .
$$

The purpose of this paper is to provide lower bounds on $H\left(x, y, z ; P_{s}\right)$ in a broader range of the parameters $y$ and $z$. We split our results according to the range of the parameter $\eta=$ $\log (z / y)$. For small values of $\eta$ lower bounds on $H\left(x, y, z ; P_{s}\right)$ depend heavily on inequalities of the form

$$
\pi(x ; q, a) \geq \frac{c x}{\phi(q) \log x} \quad \text { for } \quad(a, q)=1
$$

for some $c>0$, uniformly in some range of $q$ with a possible 'small' exceptional set, namely reverse Brun-Titchmarsh inequalities. Such results have been proven by Alford, Granville and Pomerance [1] and Harman [16]. Also, Bombieri, Friedlander and Iwaniec proved in [3] an asymptotic formula for

$$
\sum_{\substack{q \leq Q \\(q, a)=1}} \pi(x ; q, a)
$$

when $Q \leq x^{1-\epsilon}$ and $a$ is fixed. Combining these results with the arguments leading to Theorem 1.2 we show the following theorem. Here and for the rest of this paper $x_{0}(\cdot)$ 
denotes a sufficiently large positive constant which depends only on the parameters given, e.g. $x_{0}(s)$, and its meaning might change from statement to statement.

Theorem 1.5 (Small values of $\eta$ ). Fix $s \in \mathbb{Z} \backslash\{0\}$. Let $3 \leq y+1 \leq z \leq x$ with $y \leq \sqrt{x}$ and $\{y<d \leq z:(d, s)=1\} \neq \emptyset$.

(a) Let $\epsilon>0$. If $x \geq x_{0}(s, \epsilon), z \leq x^{5 / 12-\epsilon}$ and

$$
y+\log \log y \leq z \leq y+\frac{y}{(\log y)^{2}},
$$

then

$$
H\left(x, y, z ; P_{s}\right) \gg \begin{cases}\frac{H(x, y, z)}{\log x} & z \geq y+(\log y)^{2 / 3}, \\ \frac{x}{\log x} \sum_{\substack{y<d \leq z \\(d, s)=1}} \frac{1}{\phi(d)} & z \leq y+(\log y)^{2 / 3}\end{cases}
$$

with the implied constant depending on $s$ and $\epsilon$. If, in addition, $(z-y) / \log \log y \rightarrow \infty$ as $y \rightarrow \infty$, then

$$
H\left(x, y, z ; P_{s}\right) \sim_{\epsilon, s} \begin{cases}f(s) \frac{315 \zeta(3)}{2 \pi^{4}} \frac{\eta x}{\log x} \quad \text { if } \frac{z-y}{(\log y)^{2 / 3}} \rightarrow \infty, \\ \frac{x}{\log x} \sum_{\substack{y<d \leq z \\(d, s)=1}} \frac{1}{\phi(d)} \quad \text { otherwise, }\end{cases}
$$

as $y \rightarrow \infty$, where $f(s)=\prod_{p \mid s} \frac{(p-1)^{2}}{p^{2}-p+1}$.

(b) If $x \geq x_{0}(s), z \leq x^{0.472}$ and

$$
y+\exp \left\{4.532(\log y)^{1 / 4}\right\} \leq z \leq y+\frac{y}{(\log y)^{2}}
$$

then (1.4) holds with the implied constant depending on $s$.

(c) If (1.3) holds for some $c>0$, uniformly in $q \leq Q$ for some $Q=Q(x) \leq \sqrt{x}$, $x \geq x_{0}(s, c)$ and

$$
z \leq y+\frac{y}{(\log y)^{2}}
$$

then (1.4) is valid for $z \leq Q$ with the implied constant depending on $s$ and $c$.

(d) Let $B \geq 2$ be fixed. If

$$
z \geq y+\frac{y}{(\log y)^{B}} \quad \text { and } \quad \xi \rightarrow \infty
$$

then

$$
H\left(x, y, z ; P_{s}\right) \sim_{s, B} f(s) \frac{315 \zeta(3)}{2 \pi^{4}} \frac{\eta x}{\log x} \quad(y \rightarrow \infty)
$$


For intermediate and large values of $\eta$ we need results about primes in arithmetic progressions on average in order to control error terms coming from the linear sieve. The most famous such result is the Bombieri-Vinogradov theorem [4, p. 161]. This theorem allows one to get the expected order of $H\left(x, y, z ; P_{s}\right)$ for $y \leq x^{1 / 2-\epsilon}$. To go beyond this threshold we make use of Theorem 9 in [3].

Theorem 1.6 (Intermediate and large values of $\eta$; short intervals). Fix $s \in \mathbb{Z} \backslash\{0\}$ and $B \geq 2$. Let $x \geq x_{0}(s, B), x(\log x)^{-B} \leq \Delta \leq x$ and $3 \leq y+1 \leq z \leq x$ with $\{y<d \leq z$ : $(d, s)=1\} \neq \emptyset, y \leq \sqrt{x}$ and

Then

$$
z \geq y+\frac{y}{(\log y)^{B}}
$$

$$
H\left(x, y, z ; P_{s}\right)-H\left(x-\Delta, y, z ; P_{s}\right) \gg_{s, B} \frac{\Delta}{x} \frac{H(x, y, z)}{\log x} .
$$

We may combine Theorems 1.3 and 1.6 with an argument given in [11] to obtain the expected order of $H\left(x, y, z ; P_{s}\right)$ in the full range of the parameters $y$ and $z$, when $\eta \geq(\log y)^{-B}$ for some fixed $B \geq 2$.

Theorem 1.7 (Intermediate and large values of $\eta$ ). Fix $s \in \mathbb{Z} \backslash\{0\}$ and $B \geq 2$. Let $x \geq x_{0}(s, B)$ and $3 \leq y+1 \leq z \leq x$ with $\{y<d \leq z:(d, s)=1\} \neq \emptyset$ and

Then

$$
z \geq y+\frac{y}{(\log y)^{B}} \text {. }
$$

$$
H\left(x, y, z ; P_{s}\right) \asymp_{s, B} \frac{H(x, y, z)}{\log x} .
$$

Finally, when $\eta$ is very large we are able to establish an asymptotic formula for $H\left(x, y, z ; P_{s}\right)$, similar to the one given for $H(x, y, z)$ in Theorem 21(iv) of [15].

Theorem 1.8 (Very large values of $\eta$ ). Let $s \in \mathbb{Z} \backslash\{0\}$. If $2 \leq y \leq z \leq x$, then

$$
H\left(x, y, z ; P_{s}\right)=\frac{x}{\log x}\left(1+O_{s}\left(\frac{\log y}{\log z}\right)\right) .
$$

Shifted primes in the multiplication table. A straightforward application of Theorem 1.7 is to the multiplication table problem. This problem, which was first posed by Erdős [8], [9], is to count the number of distinct integers of the form $a b$ with $1 \leq a, b \leq N$, namely to estimate the quantity

$$
A(N):=|\{a b: 1 \leq a, b \leq N\}|
$$

A related question is to estimate

$$
A\left(N ; P_{s}\right):=\left|\left\{a b \in P_{s}: 1 \leq a, b \leq N\right\}\right|,
$$

that is how many shifted primes appear in the multiplication table. The order of $A(N)$ was determined by Ford in [11], where he proved that

$$
A(N) \asymp \frac{N^{2}}{(\log N)^{\delta}(\log \log N)^{3 / 2}} .
$$


This follows by the elementary inequalities

$$
H\left(\frac{N^{2}}{2}, \frac{N}{2}, N\right) \leq A(N) \leq \sum_{m \geq 0} H\left(\frac{N^{2}}{2^{m}}, \frac{N}{2^{m+1}}, \frac{N}{2^{m}}\right)
$$

and Theorem 1.1. Similarly, using Theorem 1.7we establish the order of magnitude of $A\left(N ; P_{s}\right)$.

Corollary 1.1. If $N \geq N_{0}(s)$, then

$$
A\left(N ; P_{s}\right) \asymp_{s} \frac{A(N)}{\log N} .
$$

\section{BACKGROUND MATERIAL}

Notation. We make use of some standard notation. If $a(n), b(n)$ are two arithmetic functions, then we denote with $a * b$ their Dirichlet convolution. Furthermore, for $n \in \mathbb{N}$ and $1 \leq y \leq z$ we put $\omega(n ; y, z)=\mid\{p$ prime $: p \mid n, y<p \leq z\} \mid$ and $\Omega(n ; y, z)=\sum\left\{a: p^{a} \| n, y<\right.$ $p \leq z\}$, where $p^{a} \| n$ means that $p^{a} \mid n$ and $p^{a+1} \nmid n$. Also, for brevity let $\omega(n ; z)=\omega(n ; 1, z)$ and $\Omega(n ; z)=\Omega(n ; 1, z)$. For $n \in \mathbb{N}$ we use $P^{+}(n)$ and $P^{-}(n)$ to denote the largest and smallest prime factor of $n$, respectively, with the notational conventions that $P^{+}(1)=0$ and $P^{-}(1)=+\infty$. Given $1 \leq y<z, \mathscr{P}(y, z)$ denotes the set of all integers $n$ such that $P^{+}(n) \leq z$ and $P^{-}(n)>y$. In addition, $\pi(x ; q, a)$ stands for the number of primes up to $x$ in the arithmetic progression $a(\bmod q)$. Lastly, for a Dirichlet character $\chi, N(\sigma, V, \chi)$ denotes the number of zeros $\rho=\beta+i \gamma$ of its associated $L$-function with $|\gamma| \leq V$ and $\beta \geq \sigma$.

In this section we state various preliminary results that are needed in order to prove Theorems 1.5, 1.6, 1.7 and 1.8, First, we list a series of results on primes in arithmetic progressions. We start with a lemma which is a direct corollary of Theorem 2.1 in [1].

Lemma 2.1. Let $\epsilon \in(0,1 / 12)$. There exists $x_{\epsilon} \geq 1$ such that for every $x \geq x_{\epsilon}$, there is a set $\mathcal{D}_{\epsilon}(x) \subset \mathbb{N} \cap[\log x, x]$ with $\left|\mathcal{D}_{\epsilon}(x)\right| \ll_{\epsilon} 1$ such that for every $(a, q)=1$ with $q \leq x^{5 / 12-\epsilon}$,

$$
\left|\pi(x ; q, a)-\frac{\operatorname{li}(x)}{\phi(q)}\right| \leq \epsilon \frac{\operatorname{li}(x)}{\phi(q)}
$$

with the possible exception of $q \in \mathcal{M D}_{\epsilon}(x)=\left\{m d: m \in \mathbb{N}, d \in \mathcal{D}_{\epsilon}(x)\right\}$.

Harman [16], allowing a larger set of exceptional moduli, gave a variation of Lemma 2.1. His starting point is the following result.

Lemma 2.2. Given $\epsilon>0$, there are constants $K(\epsilon) \geq 2$ and $c(\epsilon)>0$ such that if $K(\epsilon)<$ $q<x^{0.472}$ and for every $d \mid q$ with $\chi$ a primitive character $(\bmod d)$ we have

$$
L(\sigma+i t, \chi) \neq 0 \quad \text { for } \sigma>1-\frac{1}{(\log q)^{3 / 4}},|t| \leq \exp \left\{\epsilon(\log q)^{3 / 4}\right\}
$$

then for any a with $(a, q)=1$ we have

$$
\pi(x ; q, a) \geq \frac{c(\epsilon) x}{\phi(q) \log x} .
$$


Using Lemma 2.2 along with estimates on averages of $N(\sigma, V, \chi)$ Harman showed a variation of Lemma 2.1. The main part of the argument is given in [16], but the result is not stated explicitly; we state it and prove it here for the sake of completeness.

Lemma 2.3. There exist absolute positive constants $c_{1}, c_{2}$ and $x_{0}$ so that for all $x \geq x_{0}$ there is a set $\mathcal{E}(x) \subset \mathbb{N} \cap[\log x, x]$ satisfying the following:

(1) $|\mathcal{E}(x)| \leq \exp \left\{3.641(\log x)^{1 / 4}\right\}$;

(2) $\left|\mathcal{E}(x) \cap\left[1, \exp \left\{c_{1}(\log x)^{3 / 4}\right\}\right]\right| \ll 1$;

(3) For every $(a, q)=1$ with $q \leq x^{0.472}$ we have

$$
\pi(x ; q, a) \geq \frac{c_{2} x}{\phi(q) \log x},
$$

with the possible exception of $q \in \mathcal{M E}(x)=\{m e: m \in \mathbb{N}, e \in \mathcal{E}(x)\}$.

Proof. Set $W=(0.4166 \log x)^{3 / 4}$. From [4, p. 93, 95] there is an absolute constant $c_{1}$ such that there is at most one primitive character $\chi_{1}$ to a modulus $q_{1} \leq V=\exp \left\{c_{1}(\log x)^{3 / 4}\right\}$ whose $L$-function has a zero $\rho$ with $|\operatorname{Im}(\rho)| \leq V$ and $\operatorname{Re}(\rho)>1-1 / W$. By [4, p. 96], this exceptional modulus $q_{1}$ satisfies $q_{1} \geq \log x$. In addition, Montgomery showed in [20] that

$$
\sum_{q \leq Q} \sum_{\chi(\bmod q)}^{*} N(\sigma, V, \chi) \ll\left(Q^{2} V\right)^{2(1-\sigma) / \sigma}(\log Q V)^{13} \quad(4 / 5 \leq \sigma \leq 1),
$$

where $\sum^{*}$ means that the sum runs over primitive characters only. Inequality (2.1) with $Q=x^{0.472}$ and $\sigma=1-1 / W$ yields that $N(\sigma, V, \chi)=0$ for all primitive characters to every moduli $q \leq x^{0.472}$ with at most $\exp \left\{3.64094(\log x)^{1 / 4}\right\}$ exceptions. Call this exceptional set $\mathcal{E}_{1}(x)$. This set contains no elements $\leq \log x$ and at most one element $\leq V$, by the discussion in the beginning of the proof. Next, applying Lemma 2.1 with $\epsilon_{0}=2 / 3 \times 10^{-4}$ we obtain a set $\mathcal{D}_{\epsilon_{0}}(x) \subset[\log x, x]$ with boundedly many elements and the property that if $q \leq x^{0.4166}$ and $q \notin \mathcal{M} \mathcal{D}_{\epsilon_{0}}(x)$, then

$$
\pi(x ; q, a) \geq\left(1-\epsilon_{0}\right) \frac{x}{\phi(q) \log x} \quad \text { for } \quad(a, q)=1 .
$$

Set

$$
\mathcal{E}(x)=\mathcal{E}_{1}(x) \cup \mathcal{D}_{\epsilon_{0}}(x) .
$$

Clearly, conditions (1) and (2) hold for $\mathcal{E}(x)$. Also, if $q \leq x^{0.4166}$ is such that $q \notin \mathcal{M E}(x)$, then (3) holds by (2.2). Finally, if $q \in\left[x^{0.4166}, x^{0.472}\right]$ and $q \notin \mathcal{M E}(x)$, then the hypothesis of Lemma 2.2 is met and we deduce (3). This completes the proof of the lemma.

Below we state the Brun-Titchmarsh inequality [14, Theorem 3.7].

Lemma 2.4. Uniformly in $1 \leq q<y \leq x$ and $(a, q)=1$ we have that

$$
\pi(x ; q, a)-\pi(x-y ; q, a) \ll \frac{y}{\phi(q) \log (2 y / q)} .
$$


In addition, we will need a generalization of Lemma 2.4, which is an easy application of the results and methods in [22]. Let $\mathcal{M}$ denote the class of functions $F: \mathbb{N} \rightarrow[0,+\infty)$ for which there exist constants $A_{F}$ and $B_{F, \epsilon}, \epsilon>0$, such that

$$
F(n m) \leq \min \left\{A_{F}^{\Omega(m)}, B_{F, \epsilon} m^{\epsilon}\right\} F(n)
$$

for all $(m, n)=1$ and all $\epsilon>0$.

Lemma 2.5. Let $F \in \mathcal{M}, a \in \mathbb{Z} \backslash\{0\}$ and $1 \leq q \leq h \leq x$ such that $(a, q)=1$ and $x>|a|$. If $q \leq x^{1-\epsilon}$ and $\frac{h}{q} \geq\left(\frac{x-a}{q}\right)^{\epsilon}$ for some $\epsilon>0$, then

$$
\sum_{\substack{x-h<p \leq x \\ p \equiv a(\bmod q)}} F\left(\frac{p-a}{q}\right) \ll_{a, \epsilon, F} \frac{h}{\phi(q)(\log x)^{2}} \sum_{n \leq x} \frac{F(n)}{n}
$$

the implied constant depends on $F$ only via the constants $A_{F}$ and $B_{F, \alpha}, \alpha>0$.

Proof. Observe that it suffices to show the lemma for the function $\widetilde{F}$ defined for $n=2^{r} m$ with $(m, 2)=1$ by

$$
\widetilde{F}(n)=\min \left\{A_{F}^{r}, \min _{\epsilon>0}\left(B_{F, \epsilon} 2^{r \epsilon}\right)\right\} F(m)
$$

We have that $\widetilde{F} \in \mathcal{M}$ with parameters $A_{F}$ and $B_{F, \alpha}^{2}, \alpha>0$. Without loss of generality we may assume that $\widetilde{F}(1)=1$. Also, suppose that $x \geq x_{0}(\epsilon, a, F)$, where $x_{0}(a, \epsilon, F)$ is a sufficiently large constant; otherwise, the result is trivial. Put

$$
q_{1}= \begin{cases}q & \text { if } 2 \mid a q \\ 2 q, & \text { if } 2 \nmid a q,\end{cases}
$$

and let $X=(x-a) / q_{1}$ and $H=h / q_{1}$. Note that if $p \equiv a(\bmod q)$ and $p>2$, then $p \equiv a$ $\left(\bmod q_{1}\right)$. So if we set $p=q_{1} m+a$ for $p>2$, then

$$
\begin{aligned}
\sum_{\substack{x-h<p \leq x \\
p \equiv a(\bmod q)}} \widetilde{F}\left(\frac{p-a}{q}\right) & \leq \sum_{\substack{X-H<m \leq X \\
P^{-}\left(q_{1} m+a\right)>\sqrt{X}}} \widetilde{F}\left(\frac{q_{1}}{q} m\right)+\sum_{\substack{X-H<m \leq X \\
3 \leq q_{1} m+a \leq \sqrt{X}}} \widetilde{F}\left(\frac{q_{1}}{q} m\right)+O_{a, F}(1) \\
& \ll_{a, F} \sum_{\substack{X-H<m \leq X \\
P^{-}\left(q_{1} m+a\right)>\sqrt{X}}} \widetilde{F}(m)+\sum_{\substack{X-H<m \leq X \\
m \leq \sqrt{X}-a}} \widetilde{F}(m)+1,
\end{aligned}
$$

since $q_{1} / q \in\{1,2\}$ and $\widetilde{F}(2 m) \ll_{F} \widetilde{F}(m)$ for all $m \in \mathbb{N}$. Let $F_{1}(n)=\widetilde{F}(n)$ and $F_{2}(n)$ be the characteristic function of integers $n$ such that $P^{-}(n)>\sqrt{X}$. Let $Q_{1}(x)=x, Q_{2}(x)=q_{1} x+a$ and $Q=Q_{1} Q_{2}$. Also, if $P(x) \in \mathbb{Z}[x]$, then let $\rho_{P}(m)$ be the number of solution of the 
congruence $P(x) \equiv 0(\bmod m)$. By Corollary 3 in [22], we have that

$$
\begin{aligned}
\sum_{\substack{X-H<m \leq X \\
P^{-}\left(q_{1} m+a\right)>\sqrt{X}}} \widetilde{F}(m) & =\sum_{X-H<m \leq X} F_{1}(m) F_{2}\left(m q_{1}+a\right) \\
& \ll_{a, \epsilon, F} H \prod_{p \leq X}\left(1-\frac{\rho_{Q}(p)}{p}\right) \prod_{j=1}^{2} \sum_{n \leq X} \frac{F_{j}(n) \rho_{Q_{j}}(n)}{n} \\
& \ll_{a, \epsilon} \frac{h}{\phi(q)} \frac{1}{\log ^{2} x} \sum_{n \leq X} \frac{\widetilde{F}(n)}{n},
\end{aligned}
$$

since $q \leq x^{1-\epsilon}$ and the discriminant of $Q$ depends only on $a$. Also, if the sum

$$
\sum_{\substack{X-H<m \leq X \\ m \leq \sqrt{X}-a}} \widetilde{F}(m)
$$

is non-zero, then $H \geq X / 2$. In this case Corollary 3 in [22] implies that

$$
\sum_{\substack{X-H<m \leq X \\ m \leq \sqrt{X}-a}} \widetilde{F}(m) \ll_{a, \epsilon, F} \frac{\sqrt{X}}{\log X} \sum_{n \leq X} \frac{\widetilde{F}(n)}{n} \ll_{a, \epsilon} \frac{h}{q \log ^{2} x} \sum_{n \leq X} \frac{\widetilde{F}(n)}{n},
$$

which together with (2.3) completes the proof of the lemma.

Using Lemma 2.5 we prove the following estimate.

Lemma 2.6. Let $1 \leq v \leq v_{0}<2, a \in \mathbb{Z} \backslash\{0\}, 1 \leq q \leq x$ and $3 / 2 \leq y \leq(x-a) / q$ with $(a, q)=1$ and $x>|a|$. If $q \leq x^{1-\epsilon}$ for some $\epsilon>0$, then

$$
\sum_{\substack{p \leq x \\ p \equiv a(\bmod q)}} v^{\Omega\left(\frac{p-a}{q} ; y\right)} \ll_{a, \epsilon, v_{0}} \frac{x}{\phi(q) \log x}(\log y)^{v-1} .
$$

Proof. We may assume that $x \geq x_{0}\left(a, \epsilon, v_{0}\right)$, where $x_{0}\left(a, \epsilon, v_{0}\right)$ is a sufficiently large constant. Let $X=(x-a) / q$ and write $v^{\Omega(n ; y)-\omega(n ; y)}=(1 * b)(n)$, where $b$ is the multiplicative function that satisfies

$$
b\left(p^{l}\right)= \begin{cases}0 & \text { if } l=1 \text { or } p>y \\ v^{l-2}(v-1) & \text { if } l \geq 2 \text { and } p \leq y\end{cases}
$$

Then

$$
v^{\Omega(n ; y)}=v^{\omega(n ; y)} \sum_{k f=n} b(k) \leq \sum_{k f=n} b(k) v^{\omega(k ; y)} v^{\omega(f ; y)}
$$

and consequently

$$
\sum_{\substack{p \leq x \\ p \equiv a(\bmod q)}} v^{\Omega\left(\frac{p-a}{q} ; y\right)} \leq \sum_{k \leq X} v^{\omega(k ; y)} b(k) \sum_{\substack{p \leq x \\ p \equiv a(\bmod q k)}} v^{\omega\left(\frac{p-a}{q k} ; y\right)} .
$$


If $k \leq \sqrt{X}$, then $k q \leq x^{1-\epsilon / 3}$. So Lemma 2.5 implies that

$$
\sum_{\substack{p \leq x \\ p \equiv a(\bmod q k)}} v^{\omega\left(\frac{p-a}{q k} ; y\right)} \ll_{a, \epsilon} \frac{x(\log y)^{v-1}}{\phi(k q) \log x} .
$$

If $k>\sqrt{X}$, then

$$
\sum_{\substack{p \leq x \\ p \equiv a(\bmod q k)}} v^{\omega\left(\frac{p-a}{q k} ; y\right)} \leq \sum_{m \leq X / k} v^{\omega(m)} \ll_{a, \epsilon} \frac{x(\log X)^{v-1}}{k q},
$$

by Theorem 01 in [15]. Hence the right hand side of (2.4) is

$$
\ll_{a, \epsilon} \frac{x(\log y)^{v-1}}{\phi(q) \log x} \sum_{k \leq \sqrt{X}} \frac{v^{\omega(k ; y)} b(k)}{\phi(k)}+\frac{x(\log X)^{v-1}}{q X^{\alpha / 2}} \sum_{\sqrt{X}<k \leq X} \frac{v^{\omega(k ; y)} b(k) k^{\alpha}}{k} \ll_{a, \epsilon, v_{0}} \frac{x(\log y)^{v-1}}{\phi(q) \log x},
$$

provided that $0<\alpha<1 / 2$ and $2^{1-\alpha}>v_{0}$, which completes the proof.

We complete the results about primes in arithmetic progressions with the following estimate.

Lemma 2.7. Let $a \in \mathbb{Z} \backslash\{0\}, \epsilon>0$ and $A>0$. There exists $B=B(A)$ such that if $R \leq x^{1 / 10-\epsilon}$ and $Q R<x(\log x)^{-B}$, then

$$
\sum_{\substack{r \leq R \\(r, a)=1}}\left|\sum_{\substack{q \leq Q \\(q, a)=1}}\left(\pi(x ; r q, a)-\frac{\operatorname{li}(x)}{\phi(r q)}\right)\right| \ll_{a, \epsilon, A} \frac{x}{(\log x)^{A}} .
$$

Proof. Use Theorem 9 in [3] plus partial summation.

We need an estimate on the summatory function of the reciprocals of Euler's $\phi$ function and other closely related quantities. Such a result was proved by Sitaramachandra [23]. Using the methods of [23] we extend this result according to the needs of this paper.

Lemma 2.8. Let $a \in \mathbb{N}, s \in \mathbb{Z}$ and $x \geq 1$ such that $1 \leq|s| \leq x$. Then

$$
\begin{aligned}
& \sum_{\substack{n \leq x \\
(n, s)=1}} \frac{\phi(a)}{\phi(a n)}=\frac{315 \zeta(3)}{2 \pi^{4}} \frac{\phi(s)}{|s|} g(\text { as })\left(\log x+\gamma-\sum_{p \nmid a s} \frac{\log p}{p^{2}-p+1}+\sum_{p \mid s} \frac{\log p}{p-1}\right) \\
& +O\left(\tau(s) \frac{a|s|}{\phi(a s)} \frac{(\log 2 x)^{2 / 3}}{x}\right),
\end{aligned}
$$

where $g($ as $)=\prod_{p \mid a s} \frac{p(p-1)}{p^{2}-p+1}$.

Proof. Since the proof of this part is along the same lines with the proof of the main result in [23], we simply sketch it. Let $P(x)=\{x\}-1 / 2$, where $\{x\}$ denotes the fractional part of $x$. Then using the estimate

$$
\sum_{n \leq x} \frac{P(x / n)}{n} \ll(\log 2 x)^{2 / 3}
$$


which was proved in [27, p. 98], along with an argument similar to the one leading to Lemma 2.2 in [23], we find that

$$
\sum_{\substack{n \leq x \\(n, m)=1}} \frac{\mu^{2}(n)}{\phi(n)} P(x / n) \ll \frac{|m|}{\phi(m)}(\log 2 x)^{2 / 3}
$$

for every $m \in \mathbb{Z} \backslash\{0\}$. Also, by the Euler-McLaurin's summation formula we have

$$
\sum_{n \leq x} \frac{1}{n}=\log x+\gamma-\frac{P(x)}{x}+O\left(\frac{1}{x^{2}}\right) .
$$

Observe that the arithmetic function $n \rightarrow \phi(a) / \phi(a n)$ is multiplicative. In particular, we have that

$$
\frac{\phi(a)}{\phi(a n)}=\sum_{\substack{k f=n \\(k, a)=1}} \frac{\mu^{2}(k)}{k \phi(k) f} .
$$

Using relations (2.5), (2.6) and (2.7) and estimating the error terms as in [23] gives us that

$$
\begin{aligned}
\sum_{\substack{n \leq x \\
(n, s)=1}} \frac{\phi(a)}{\phi(a n)} & =\sum_{\substack{k \leq x \\
(k, a s)=1}} \frac{\mu^{2}(k)}{k \phi(k)} \sum_{\substack{f \leq x / k \\
(f, s)=1}} \frac{1}{f}=\sum_{d \mid s} \frac{\mu(d)}{d} \sum_{\substack{k \leq x / d \\
(k, a s)=1}} \frac{\mu^{2}(k)}{k \phi(k)} \sum_{l \leq x / k d} \frac{1}{l} \\
& =\sum_{d \mid s} \frac{\mu(d)}{d} \sum_{\substack{k \leq x / d \\
(k, a s)=1}} \frac{\mu^{2}(k)}{k \phi(k)}\left(\log \frac{x / d}{k}+\gamma-\frac{k}{x / d} P\left(\frac{x / d}{k}\right)+O\left(\frac{k^{2}}{(x / d)^{2}}\right)\right) \\
& =\sum_{d \mid s} \frac{\mu(d)}{d} \sum_{\substack{k=1 \\
(k, a s)=1}}^{\infty} \frac{\mu^{2}(k)}{k \phi(k)}\left(\log \frac{x / d}{k}+\gamma\right)+O\left(\frac{\tau(s) a|s|}{\phi(a s)} \frac{(\log 2 x)^{2 / 3}}{x}\right),
\end{aligned}
$$

since $|s| \leq x$. Finally, a simple calculation and the identity

$$
\sum_{k=1}^{\infty} \frac{\mu^{2}(k)}{k \phi(k)}=\frac{315 \zeta(3)}{2 \pi^{4}}
$$

complete the proof.

The following result is known as the 'fundamental lemma' of sieve methods. It has appeared in the literature in several different forms (see for example [14, Theorem 2.5, p. 82]). We need a version of it that can be found in [13] and [19].

Lemma 2.9. Let $D \geq 2, D=Z^{v}$ with $v \geq 3$.

(a) Fix $\kappa>0$. There exist two sequences $\left\{\lambda^{+}(d)\right\}_{d \leq D}$, and $\left\{\lambda^{-}(d)\right\}_{d \leq D}$ such that

$$
\begin{gathered}
\left|\lambda^{ \pm}(d)\right| \leq 1, \\
\begin{cases}\left(\lambda^{-} * 1\right)(n)=\left(\lambda^{+} * 1\right)(n)=1 & \text { if } P^{-}(n)>Z, \\
\left(\lambda^{-} * 1\right)(n) \leq 0 \leq\left(\lambda^{+} * 1\right)(n) & \text { otherwise, }\end{cases}
\end{gathered}
$$


and, for any multiplicative function $\alpha(d)$ with $0 \leq \alpha(p) \leq \min \{\kappa, p-1\}$,

$$
\sum_{d \leq D} \lambda^{ \pm}(d) \frac{\alpha(d)}{d}=\prod_{p \leq Z}\left(1-\frac{\alpha(p)}{p}\right)\left(1+O_{\kappa}\left(e^{-v}\right)\right) .
$$

(b) There exists a sequence $\{\rho(d)\}_{d \leq D}$ such that

$$
\begin{gathered}
|\rho(d)| \leq 1, \\
\begin{cases}(\rho * 1)(n)=1 & \text { if } P^{-}(n)>Z, \\
(\rho * 1)(n) \leq 0 & \text { otherwise, }\end{cases}
\end{gathered}
$$

and, for any multiplicative function $\alpha(d)$ satisfying $0 \leq \alpha(p) \leq p-1$ and

$$
\prod_{y<p \leq w}\left(1-\frac{\alpha(p)}{p}\right)^{-1} \leq \frac{\log w}{\log y}\left(1+\frac{L}{\log y}\right) \quad(3 / 2 \leq y \leq w)
$$

we have

$$
\sum_{d \leq D} \rho(d) \frac{\alpha(d)}{d} \gg \prod_{p \leq Z}\left(1-\frac{\alpha(p)}{p}\right),
$$

provided that $D \geq D_{0}(L)$, where $D_{0}(L)$ is a constant depending only on $L$.

Proof. (a) The result follows by [13, Lemma 5, p. 732].

(b) The construction of the sequence $\{\rho(d)\}_{d \leq D}$ and the proof that it satisfies the desired properties is based on [13, Lemma 5] and [19, Lemma 3]. We sketch the proof below. Without loss of generality we may assume that $Z \notin \mathbb{N}$. Set $P(Z)=\prod_{p<Z} p$ and $\rho(d)=\mu(d) \mathbf{1}_{A}(d)$, where $\mathbf{1}_{A}$ is the characteristic function of the set

$$
A=\left\{d \mid P(Z): d=p_{1} \cdots p_{r}, p_{r}<\cdots<p_{1}<Z, p_{2 l}^{3} p_{2 l-1} \cdots p_{1}<D(1 \leq l \leq r / 2)\right\} .
$$

By the proof of Lemma 5 in [13], the sequence $\{\rho(d)\}_{d=1}^{\infty}$ is supported in $\{d \in \mathbb{N}: d<D\}$ and satisfies (2.8) and (2.9). Finally, by Lemma 3 in [19], there exists a function $h$, independent of the parameters $D, Z$ and $L$, such that

$$
\sum_{d \leq D} \rho(d) \frac{\alpha(d)}{d} \geq\left(h(v)+O\left(e^{\sqrt{L}-v}(\log D)^{-1 / 3}\right)\right) \prod_{p<Z}\left(1-\frac{\alpha(p)}{p}\right)
$$

for all multiplicative functions $\alpha(d)$ that satisfy $0 \leq \alpha(p) \leq p-1$ and (2.10). In addition, $h$ is increasing and $h(3)>0$, by [18, p. 172-173]. This proves that (2.11) holds too and completes the proof of the lemma.

We now introduce some notation we will be utilizing later. For $a$ and $k$ in $\mathbb{N}$ and $1 \leq y<z$ define

$$
\tau(a)=|\{d \in \mathbb{N}: d \mid n\}|, \quad \tau(a, y, z)=|\{d \in \mathbb{N}: d \mid n, y<d \leq z\}|
$$

and

$$
\tau_{k}(a)=\left|\left\{\left(d_{1}, \ldots, d_{k}\right) \in \mathbb{N}^{k}: d_{1} \cdots d_{k}=a\right\}\right|
$$


Moreover, for $\sigma>0$ let

$$
\mathscr{L}(a ; \sigma)=\left\{x \in \mathbb{R}: \tau\left(a, e^{x}, e^{x+\sigma}\right) \geq 1\right\}=\bigcup_{d \mid a}[\log d-\sigma, \log d)
$$

and

$$
L(a ; \sigma)=\operatorname{meas}(\mathscr{L}(a ; \sigma)),
$$

where 'meas' denotes the Lebesgue measure on the real line. We note the straightforward inequality

$$
L(a b ; \sigma) \leq \tau(a) L(b ; \sigma) \quad \text { for } \quad(a, b)=1,
$$

which is item (ii) of Lemma 3.1 in [11.

When $\eta$ is in the intermediate range of values, the basic result we will use to bound $H\left(x, y, z ; P_{s}\right)$ from below is the following estimate.

Lemma 2.10. Let $\epsilon>0, B>0, x \geq 1,3 \leq y+1 \leq z$ with $z \leq x^{2 / 3}$ and $\eta \in\left[(\log y)^{-B}, \frac{\log y}{100}\right]$. Then

$$
H(x, y, z) \asymp_{\epsilon, B} \frac{x}{\log ^{2} y} \sum_{\substack{a \leq y^{\epsilon} \\ \mu^{2}(a)=1}} \frac{L(a ; \eta)}{a} .
$$

The proof of Lemma 2.10 can be found in [11]. Even though this result is not stated explicitly, it is a direct corollary of the methods there: see Theorem 1 and Lemmas 4.1, 4.2, 4.5, 4.8 and 4.9 in [11]. Also, we will need the following result, which is Corollary 1 in [11].

Lemma 2.11. Suppose $x_{1}, y_{1}, z_{1}, x_{2}, y_{2}, z_{2}$ are real numbers with $2 \leq y_{i}+1 \leq z_{i} \leq x_{i}(i=$ $1,2), \log \left(z_{1} / y_{1}\right) \asymp \log \left(z_{2} / y_{2}\right), \log y_{1} \asymp \log y_{2}$ and $\log \left(x_{1} / z_{1}\right) \asymp \log \left(x_{2} / z_{2}\right)$. Then

$$
\frac{H\left(x_{1}, y_{1}, z_{1}\right)}{x_{1}} \asymp \frac{H\left(x_{2}, y_{2}, z_{2}\right)}{x_{2}} .
$$

Finally, we state a covering lemma, which a special case of Lemma 3.15 in [10]. Here for $I$ an interval of the real line we denote by $r I$ the interval that has the same center as $I$ and $r$ times its diameter.

Lemma 2.12. Let $A=\bigcup_{n=1}^{N} I_{n} \subset \mathbb{R}$, where the sets $I_{n}$ are nonempty intervals of the form $[a, b)$. Then there exists a subcollection $\left\{I_{i_{m}}\right\}_{m=1}^{M}$ of mutually disjoint intervals such that

$$
A \subset \bigcup_{m=1}^{M} 3 I_{i_{m}}
$$

\section{Small values of $\eta$}

In this section we give the proof of Theorem 1.5. First, we show an auxiliary result.

Lemma 3.1. Let $a \in \mathbb{Z} \backslash\{0\}, x \geq 2$ and $3 \leq Q_{1}+1 \leq Q_{2} \leq 2 Q_{1}$ with $Q_{1} \leq \sqrt{x}$ and $\left\{Q_{1}<q \leq Q_{2}:(q, a)=1\right\} \neq \emptyset$. 
(a) Let $\epsilon \in(0,1 / 12)$. If $x \geq x_{0}(a, \epsilon)$ and $Q_{1}+\log \log Q_{1} \leq Q_{2} \leq x^{5 / 12-\epsilon}$, then

$$
\sum_{\substack{Q_{1}<q \leq Q_{2} \\(q, a)=1}} \pi(x ; q, a) \gg_{a, \epsilon} \frac{x}{\log x} \sum_{\substack{Q_{1}<q \leq Q_{2} \\(q, a)=1}} \frac{1}{\phi(q)} .
$$

If, in addition, $\left(Q_{2}-Q_{1}\right) / \log \log Q_{1} \rightarrow \infty$ as $Q_{1} \rightarrow \infty$, then

$$
\sum_{\substack{Q_{1}<q \leq Q_{2} \\(q, a)=1}} \pi(x ; q, a) \sim_{a, \epsilon} \frac{x}{\log x} \sum_{\substack{Q_{1}<q \leq Q_{2} \\(q, a)=1}} \frac{1}{\phi(q)} \quad\left(Q_{1} \rightarrow \infty\right) .
$$

(b) If $x \geq x_{0}(a)$ and $Q_{1}+\exp \left\{4.532\left(\log Q_{1}\right)^{1 / 4}\right\} \leq Q_{2} \leq x^{0.472}$, then (3.1) holds with the implied constant depending only on $a$.

(c) Let $B \geq 2$. If $Q_{2} \geq Q_{1}+Q_{1}\left(\log Q_{1}\right)^{-B}$, then

$$
\sum_{\substack{Q_{1}<q \leq Q_{2} \\(q, a)=1}} \pi(x ; q, a) \sim_{a, B} f(a) \frac{315 \zeta(3)}{2 \pi^{4}} \frac{x \log \left(Q_{2} / Q_{1}\right)}{\log x} .
$$

Proof. (a) For every $\epsilon_{1} \in(0, \epsilon]$ and $x \geq x_{\epsilon_{1}}$ Lemmas 2.1 and 2.4 imply that

$$
\begin{aligned}
\sum_{\substack{Q_{1}<q \leq Q_{2} \\
(q, a)=1}} \pi(x ; q, a) & =\left(1+\epsilon_{1} \theta\right) \operatorname{li}(x) \sum_{\substack{Q_{1}<q \leq Q_{2} \\
q \notin \mathcal{M} \mathcal{D}_{\epsilon_{1}}(x) \\
(q, a)=1}} \frac{1}{\phi(q)}+O\left(\frac{x}{\log x} \sum_{\substack{Q_{1}<q \leq Q_{2} \\
q \in \mathcal{M} \mathcal{D}_{\epsilon_{1}}(x)}} \frac{1}{\phi(q)}\right) \\
& =\left(1+\epsilon_{1} \theta\right) \operatorname{li}(x) \sum_{\substack{Q_{1}<q \leq Q_{2} \\
(q, a)=1}} \frac{1}{\phi(q)}+O\left(\frac{x}{\log x} \sum_{\substack{Q_{1}<q \leq Q_{2} \\
q \in \mathcal{M} \mathcal{D}_{\epsilon_{1}}(x)}} \frac{1}{\phi(q)}\right),
\end{aligned}
$$

for some $|\theta| \leq 1$. Fix $d \in \mathcal{D}_{\epsilon_{1}}(x)$. If $d \geq Q_{2}-Q_{1}$, then the interval $\left(Q_{1} / d, Q_{2} / d\right]$ contains at most one integer and therefore

$$
\sum_{Q_{1} / d<m \leq Q_{2} / d} \frac{1}{\phi(d m)} \ll \frac{\log \log Q_{1}}{Q_{1}} .
$$

On the other hand, if $d \leq Q_{2}-Q_{1}$, then

$$
\sum_{Q_{1} / d<m \leq Q_{2} / d} \frac{1}{\phi(d m)} \ll \frac{\log \log Q_{1}}{d} \log \left(Q_{2} / Q_{1}\right) .
$$

Since $d \geq \log x$ and $\left|\mathcal{D}_{\epsilon_{1}}(x)\right| \ll_{\epsilon_{1}} 1$, relations (3.4) and (3.5) yield that

$$
\sum_{\substack{Q_{1}<q \leq Q_{2} \\ q \in \mathcal{M} \mathcal{D}_{\epsilon_{1}}(x)}} \frac{1}{\phi(q)} \ll_{\epsilon_{1}} \frac{\log \log Q_{1}}{Q_{1}}+\frac{\log \log Q_{1}}{\log x} \log \left(Q_{2} / Q_{1}\right) .
$$

Also,

$$
\sum_{\substack{Q_{1}<q \leq Q_{2} \\(q, a)=1}} \frac{1}{\phi(q)} \geq \sum_{\substack{Q_{1}<q \leq Q_{2} \\(q, a)=1}} \frac{1}{q} \gg_{a} \log \left(Q_{2} / Q_{1}\right) \asymp \frac{Q_{2}-Q_{1}}{Q_{1}}
$$


uniformly in $Q_{1}+1 \leq Q_{2} \leq 2 Q_{1}$ with $\left\{Q_{1}<q \leq Q_{2}:(q, a)=1\right\} \neq \emptyset$. The above inequality, (3.3) and (3.6) imply that

$$
\sum_{\substack{Q_{1}<q \leq Q_{2} \\(q, a)=1}} \pi(x ; q, a)=\left(1+\epsilon_{1} \theta\right) \operatorname{li}(x) \sum_{\substack{Q_{1}<q \leq Q_{2} \\(q, a)=1}} \frac{1}{\phi(q)}\left(1+O_{a, \epsilon_{1}}\left(\frac{\log \log Q_{1}}{\log x}+\frac{\log \log Q_{1}}{Q_{2}-Q_{1}}\right)\right) .
$$

This proves that (3.2) holds. Next, we show that (3.1) holds. Fix a large positive constant $M=M(\epsilon, a)$ with $M \geq x_{\epsilon}$. If $Q_{1} \leq M$ and $x$ is large enough, then

$$
\sum_{\substack{Q_{1}<q \leq Q_{2} \\(q, a)=1}} \pi(x ; q, a) \geq \max _{\substack{Q_{1}<q \leq Q_{2} \\(q, a)=1}} \pi(x ; q, a) \gg_{a, \epsilon} \frac{x}{\log x} \asymp_{a, \epsilon} \frac{x}{\log x} \sum_{\substack{Q_{1}<q \leq Q_{2} \\(q, a)=1}} \frac{1}{\phi(q)},
$$

by our assumption that $\left\{Q_{1}<q \leq Q_{2}:(q, a)=1\right\} \neq \emptyset$ and the Prime Number Theorem for arithmetic progressions [4, p. 123]. So we may suppose that $Q_{1}>M$. By (3.3), (3.6) and (3.7) with $\epsilon_{1}=\epsilon$ we deduce that

$$
\sum_{\substack{Q_{1}<q \leq Q_{2} \\(q, a)=1}} \pi(x ; q, a) \geq \frac{x}{2 \log x}\left(\sum_{\substack{Q_{1}<q \leq Q_{2} \\(q, a)=1}} \frac{1}{\phi(q)}-C_{a, \epsilon} \frac{\log \log Q_{1}}{Q_{1}}\right)
$$

for some positive constant $C_{a, \epsilon}$. We separate two cases. If

$$
\sum_{\substack{Q_{1}<q \leq Q_{2} \\(q, a)=1}} \frac{1}{\phi(q)} \geq 2 C_{a, \epsilon} \frac{\log \log Q_{1}}{Q_{1}}
$$

then (3.1) holds by (3.8). So assume that (3.9) fails. Then, by (3.7) and our assumption that $Q_{2} \geq Q_{1}+\log \log Q_{1}$, we have that

$$
\frac{\log \log Q_{1}}{Q_{1}} \ll \log \frac{Q_{2}}{Q_{1}} \ll_{a} \sum_{\substack{Q_{1}<q \leq Q_{2} \\(q, a)=1}} \frac{1}{q} \leq \sum_{\substack{Q_{1}<q \leq Q_{2} \\(q, a)=1}} \frac{1}{\phi(q)} \leq 2 C_{a, \epsilon} \frac{\log \log Q_{1}}{Q_{1}} .
$$

Also, Lemma 2.1 implies that

$$
\sum_{\substack{Q_{1}<q \leq Q_{2} \\(q, a)=1}} \pi(x ; q, a) \geq \frac{x}{2 \log x} \sum_{\substack{Q_{1}<q \leq Q_{2} \\(q, a)=1, q \notin \mathcal{M} \mathcal{D}_{\epsilon}(x)}} \frac{1}{\phi(q)} \geq \frac{x}{2 \log x}\left(\sum_{\substack{Q_{1}<q \leq Q_{2} \\(q, a)=1}} \frac{1}{q}-\sum_{\substack{Q_{1}<q \leq Q_{2} \\ q \in \mathcal{M} \mathcal{D}_{\epsilon}(x)}} \frac{1}{q}\right)
$$

By the argument leading to (3.6) we find that

$$
\sum_{\substack{Q_{1}<q \leq Q_{2} \\ q \in \mathcal{M} \mathcal{D}_{\epsilon}(x)}} \frac{1}{q} \ll_{\epsilon} \frac{1}{Q_{1}}+\frac{\log \left(Q_{2} / Q_{1}\right)}{\log x}
$$

Inserting (3.10) and (3.12) into (3.11) proves (3.1) in the case that (3.9) does not hold too.

(b) When $Q_{1} \leq x^{0.41666}<x^{5 / 12}$ the result follows from part (a). When $Q_{1}>x^{0.41666}$ note that

$$
Q_{2}-Q_{1} \geq \exp \left\{4.532\left(\log Q_{1}\right)^{1 / 4}\right\} \geq \exp \left\{3.6411(\log x)^{1 / 4}\right\}
$$


So following a very similar argument with the one given in part (a) and using Lemma 2.3 in place of Lemma 2.1 we obtain the desired result.

(c) Apply Lemmas 2.7 and 2.8.

We are now in position to prove Theorem 1.5.

Proof of Theorem 1.5. First, assume that $z \leq y+y(\log y)^{-2}$. We treat all four parts of the theorem simultaneously. Let $y_{0}$ be a large constant, possibly depending on $s, B, \epsilon$ and $c$, the constant in (1.3), according to the assumptions of each of the parts (a), (b) and (c). If $y \leq y_{0}$, then we trivially have that

$$
H\left(x, y, z ; P_{s}\right) \geq \max _{\substack{y<d \leq z \\(d, s)=1}} \pi(x-s ; d,-s) \asymp y_{0} \frac{x}{\log x},
$$

by our assumption that $\{y<d \leq z:(d, s)=1\} \neq \emptyset$ and the Prime Number Theorem for arithmetic progressions [4, p. 123]. So assume that $y>y_{0}$. By the inclusion-exclusion principle, we have that

$$
\sum_{y<d \leq z} \pi(x-s ; d,-s)-\sum_{y<d_{1}<d_{2} \leq z} \pi\left(x-s ;\left[d_{1}, d_{2}\right],-s\right) \leq H\left(x, y, z ; P_{s}\right) \leq \sum_{y<d \leq z} \pi(x-s ; d,-s) .
$$

Lemma 2.4 then implies that

$$
H\left(x, y, z ; P_{s}\right)=\sum_{\substack{y<d \leq z \\(d, s)=1}} \pi(x-s ; d,-s)+O\left(\sum_{y<d_{1}<d_{2} \leq z} \frac{x}{\log \left(2 x /\left[d_{1}, d_{2}\right]\right) \phi\left(\left[d_{1}, d_{2}\right]\right)}\right) .
$$

In the sum over $d_{1}$ and $d_{2}$ in the right hand side of (3.14) set $m=\left(d_{1}, d_{2}\right)$ and $d_{i}=m t_{i}$, $i=1,2$. Since $t_{1}+1 \leq t_{2}$, we get that $m \leq d_{2}-d_{1} \leq z-y$. Moreover, notice that

$$
\log \frac{2 x}{\left[d_{1}, d_{2}\right]}=\log \frac{2 x}{t_{1} t_{2} m} \geq \log \frac{2 x m}{z^{2}} \gg \frac{\log 2 m \log x}{\log y},
$$

uniformly in $y \leq \sqrt{x}$. Therefore

$$
\begin{aligned}
\sum_{y<d_{1}<d_{2} \leq z} \frac{1}{\log \left(2 x /\left[d_{1}, d_{2}\right]\right) \phi\left(\left[d_{1}, d_{2}\right]\right)} & \ll \frac{(\log y)(\log \log y)}{\log x} \sum_{m \leq z-y} \frac{1}{m \log 2 m} \sum_{y / m<t_{1}<t_{2} \leq z / m} \frac{1}{t_{1} t_{2}} \\
& \leq \frac{(\log y)(\log \log y)}{\log x} \sum_{m \leq z-y} \frac{1}{m \log 2 m}\left(\sum_{y / m<t \leq z / m} \frac{1}{t}\right)^{2} \\
& \ll \frac{\eta^{2}(\log y)(\log \log y)^{2}}{\log x} \ll \frac{\eta}{\log x} \frac{(\log \log y)^{2}}{\log y},
\end{aligned}
$$

which combined with (3.14) yields that

$$
H\left(x, y, z ; P_{s}\right)=\sum_{\substack{y<d \leq z \\(d, s)=1}} \pi(x-s ; d,-s)+O_{s}\left(\frac{\eta x}{\log x} \frac{(\log \log y)^{2}}{\log y}\right) .
$$


The above estimate together with Lemma 3.1 and the inequality

$$
\sum_{\substack{y<d \leq z \\(d, s)=1}} \frac{1}{\phi(d)} \geq \sum_{\substack{y<d \leq z \\(d, s)=1}} \frac{1}{d} \gg_{s} \eta
$$

which holds uniformly in $y+1 \leq z$ with $\{y<d \leq z:(d, s)=1\} \neq \emptyset$, completes the proof of parts (a), (b) and (c) as well as of part (d) when $z \leq y+y(\log y)^{-2}$. It remains to show part (d) when $z>y+y(\log y)^{-2}$, in which case $(\log y)^{-2} \ll \eta \ll(\log y)^{-\log 4+1}$. First, by (3.13) and Lemma 3.1(c), we have that

$$
H\left(x, y, z ; P_{s}\right) \leq \sum_{\substack{y<d \leq z \\(d, s)=1}} \pi(x-s ; d,-s)+O_{s}(1) \sim_{s} f(s) \frac{315 \zeta(3)}{2 \pi^{4}} \frac{\eta x}{\log x}
$$

which proves the desired upper bound. For the lower bound, let $\chi$ be the characteristic function of integers $n$ satisfying

$$
\Omega(n ; y) \leq L(y):=2 \log \log y+\psi(y)(\log \log y)^{1 / 2},
$$

where $\psi(y) \rightarrow \infty$ as $y \rightarrow \infty$ and $\psi(y) \ll(\log \log y)^{1 / 6}$. Then the inclusion-exclusion principle and Lemma 3.1 (c) imply that

$$
\begin{aligned}
H\left(x, y, z ; P_{s}\right) \geq \sum_{\substack{p+s \leq x \\
\tau(p+s, y, z) \geq 1}} \chi(p+s) & \geq \sum_{p+s \leq x} \chi(p+s)\left(\sum_{\substack{d \mid p+s \\
y<d \leq z}} 1-\sum_{\substack{\left[d_{1}, d_{2}\right] \mid p+s \\
y<d_{1}<d_{2} \leq z}} 1\right) \\
& \geq f(s) \frac{315 \zeta(3)}{2 \pi^{4}} \frac{\eta x}{\log x}\left(1-o_{s}(1)\right)-S-S^{\prime}
\end{aligned}
$$

where

$$
S:=\sum_{\substack{p+s \leq x \\ p \nmid s}}(1-\chi(p+s)) \sum_{\substack{d \mid p+s \\ y<d \leq z}} 1 \text { and } \quad S^{\prime}:=\sum_{\substack{p+s \leq x \\ p \nmid s}} \chi(p+s) \sum_{\substack{\left[d_{1}, d_{2}\right] \mid p+s \\ y<d_{1}<d_{2} \leq z}} 1 .
$$

To bound $S$ observe that for every $1 \leq v \leq 3 / 2$ we have that

$$
\begin{aligned}
S \leq v^{-L(y)} \sum_{\substack{p+s \leq x \\
p \nmid s}} v^{\Omega(p+s ; y)} \sum_{\substack{d \mid p+s \\
y<d \leq z}} 1 & \leq v^{-L(y)} \sum_{\substack{y<d \leq z \\
(d, s)=1}} v^{\Omega(d ; y)} \sum_{\substack{p+s \leq x \\
p \equiv-s(\bmod d)}} v^{\Omega\left(\frac{p+s}{d} ; y\right)} \\
& \ll_{s} \frac{x v^{-L(y)}(\log y)^{v-1}}{\log x} \sum_{y<d \leq z} \frac{v^{\Omega(d)}}{\phi(d)},
\end{aligned}
$$

by Lemma 2.6. Writing

$$
\frac{d}{\phi(d)}=\sum_{k \mid d} \frac{\mu^{2}(k)}{\phi(k)}
$$


and using Theorem 04 in [15] we find that

$$
\begin{aligned}
& \sum_{y<d \leq z} \frac{v^{\Omega(d)}}{\phi(d)}= \sum_{k \leq z} \frac{\mu^{2}(k) v^{\Omega(k)}}{k \phi(k)} \sum_{y / k<f \leq z / k} \frac{v^{\Omega(f)}}{f} \\
& \ll \sum_{k \leq \sqrt{y}} \frac{\mu^{2}(k) v^{\Omega(k)}}{k \phi(k)}\left(\eta(\log (y / k))^{v-1}+(\log (y / k))^{v-3}\right) \\
&+\sum_{\sqrt{y}<k \leq z} \frac{\mu^{2}(k) v^{\Omega(k)}}{k \phi(k)}(\log y)^{v-1} \\
& \ll \eta(\log y)^{v-1}+\frac{(\log y)^{v-1}}{y^{1 / 4}} \sum_{\sqrt{y<k \leq z}} \frac{\mu^{2}(k) v^{\Omega(k)}}{\sqrt{k} \phi(k)} \\
& \ll \eta(\log y)^{v-1},
\end{aligned}
$$

since $\eta \gg(\log y)^{-2}$. Combining inequalities (3.16) and (3.17) we find that

$$
S \ll_{s} \frac{\eta x}{\log x} \frac{(\log y)^{2 v-2}}{v^{L(y)}} .
$$

Setting $v=L(y) / 2 \log \log y$ we deduce that

$$
S \ll_{s} \frac{\eta x}{\log x} \exp \left\{-\frac{\psi(y)^{2}}{4}+O\left(\frac{\psi(y)^{3}}{(\log \log y)^{1 / 2}}\right)\right\}=o\left(\frac{\eta x}{\log x}\right) \quad(y \rightarrow \infty) .
$$

Next, we turn to the estimation of $S^{\prime}$. Note that for every $1 / 10 \leq v \leq 1$ we have that

$$
\begin{aligned}
S^{\prime} & \leq v^{-L(y)} \sum_{\substack{p+s \leq x \\
p \nmid s}} v^{\Omega(p+s ; y)} \sum_{\substack{\left[d_{1}, d_{2}\right] \mid p+s \\
y<d_{1}<d_{2} \leq z}} 1 \\
& =v^{-L(y)} \sum_{\substack{y<d_{1}<d_{2} \leq z \\
\left(d_{1} d_{2}, s\right)=1}} v^{\Omega\left(\left[d_{1}, d_{2}\right] ; y\right)} \sum_{\substack{p+s \leq x, p \nmid s \\
p \equiv-s\left(\bmod \left[d_{1}, d_{2}\right]\right)}} v^{\Omega\left(\frac{p+s}{\left[d_{1}, d_{2}\right]} ; y\right)} .
\end{aligned}
$$

Set

$$
S_{1}^{\prime}=\sum_{\substack{y<d_{1}<d_{2} \leq z \\\left(d_{1} d_{2}, s\right)=1 \\\left(d_{1}, d_{2}\right)>y^{2} x^{-3 / 4}}} v^{\Omega\left(\left[d_{1}, d_{2}\right] ; y\right)} \sum_{\substack{p+s \leq x, p \nmid s \\ p \equiv-s\left(\bmod \left[d_{1}, d_{2}\right]\right)}} v^{\Omega\left(\frac{p+s}{\left[d_{1}, d_{2}\right]} ; y\right)}
$$

and

$$
S_{2}^{\prime}=\sum_{\substack{y<d_{1}<d_{2} \leq z \\\left(d_{1} d_{2}, s\right)=1 \\\left(d_{1}, d_{2}\right) \leq y^{2} x^{-3 / 4}}} v^{\Omega\left(\left[d_{1}, d_{2}\right] ; y\right)} \sum_{\substack{p+s \leq x, p \nmid s \\ p \equiv-s\left(\bmod \left[d_{1}, d_{2}\right]\right)}} v^{\Omega\left(\frac{p+s}{\left[d_{1}, d_{2}\right]} ; y\right)} .
$$

Put $m=\left(d_{1}, d_{2}\right)$ and $d_{i}=m t_{i}$ so that $\left[d_{1}, d_{2}\right]=m t_{1} t_{2}$. Note that $m \leq z-y$. First, we deal with $S_{1}^{\prime}$. Since $v^{\Omega(n ; y)} \leq v^{\omega(n ; y)}$ for $v \leq 1$ and $\left[d_{1}, d_{2}\right] \leq 2 x^{3 / 4}$ in the range of $S_{1}^{\prime}$, Lemma 
2.5 gives us that

$$
\begin{aligned}
S_{1}^{\prime} & \ll_{s} \sum_{y^{2} x^{-3 / 4}<m \leq z-y} \sum_{y / m<t_{1}<t_{2} \leq z / m} \frac{v^{\Omega\left(m t_{1} t_{2} ; y\right)} x(\log y)^{v-1}}{\phi\left(m t_{1} t_{2}\right) \log x} \\
& \ll \frac{x(\log y)^{v-1} \log \log y}{\log x} \sum_{m \leq z-y} \frac{v^{\Omega(m)}}{m}\left(\sum_{y / m<t \leq z / m} \frac{v^{\Omega(t)}}{t}\right)^{2},
\end{aligned}
$$

uniformly in $1 / 10 \leq v \leq 1$, since $\Omega(n ; y) \geq \Omega(n)-2$ for $n \leq y^{3}$. By relation (2.39) in [15] we have

$$
\sum_{y / m<t \leq z / m} \frac{v^{\Omega(t)}}{t} \ll \eta\left(\log \frac{1}{\eta}\right)^{1-v}\left(\log \frac{y}{m}\right)^{v-1} \asymp \eta(\log \log y)^{1-v}\left(\log \frac{y}{m}\right)^{v-1},
$$

which, combined with (3.20), yields that

$$
S_{1}^{\prime} \ll_{s} \frac{\eta^{2} x(\log y)^{v-1}(\log \log y)^{3-2 v}}{\log x} \sum_{m \leq z-y} \frac{v^{\Omega(m)}}{m}\left(\log \frac{y}{m}\right)^{2 v-2} .
$$

We now estimate $S_{2}^{\prime}$. First, for $d_{1}, d_{2}$ in the range of summation of $S_{2}^{\prime}$ we have $x\left(d_{1}, d_{2}\right) / y^{2} \leq$ $x^{1 / 4}$, by definition. So if $S_{2}^{\prime}$ is a non-empty sum, we must have that $y \geq x^{3 / 8}$ and $m=$ $\left(d_{1}, d_{2}\right) \leq x^{1 / 4} \leq y^{2 / 3}$. Consequently,

$$
S_{2}^{\prime} \leq \sum_{\substack{m \leq y^{2 / 3} \\(m, s)=1}} \sum_{y / m<t_{1}<t_{2} \leq z / m} \sum_{\substack{p+s \leq x, p \nmid s \\\left(t_{1} t_{2}, s\right)=1}} v^{\Omega(p+s ; y)} .
$$

Set $p+s=m t_{1} t_{2} k$. Then we have that $k \leq x /\left(y t_{1}\right), \frac{z-y}{m} \geq\left(\frac{z}{m}\right)^{1 / 2}$ and $m t_{1} k \leq\left(t_{1} k z\right)^{7 / 8}$. Also, note that $\Omega(n ; y) \geq \Omega(n)-2$ for $n \leq x$, since $y \geq x^{3 / 8}$. So

$$
\begin{aligned}
S_{2}^{\prime} & \leq \frac{1}{v^{2}} \sum_{\substack{m \leq y^{2 / 3} \\
(m, s)=1}} \sum_{\substack{y / m<t_{1} \leq z / m \\
\left(t_{1}, s\right)=1}} \sum_{\substack{k \leq x /\left(y t_{1}\right) \\
(k, s)=1}} v^{\Omega\left(m t_{1} k\right)} \sum_{\substack{t_{1} k y<p+s \leq t_{1} k z \\
p \equiv-s\left(\bmod m t_{1} k\right)}} v^{\omega\left(\frac{p+s}{m t_{1} k}\right)} \\
& \ll \sum_{s \leq y^{2 / 3}} \sum_{y / m<t_{1} \leq z / m} \frac{v^{\Omega\left(m t_{1} k\right)} t_{1} k(z-y)\left(\log \left(t_{1} k z\right)\right)^{v-2}}{\phi\left(m t_{1} k\right)} \\
& \ll \frac{\eta y(\log y)^{v-1} \log \log y}{\log x} \sum_{m \leq y^{2 / 3}} \frac{v^{\Omega(m)}}{m} \sum_{y / m<t_{1} \leq z / m} v^{\Omega\left(t_{1}\right)} \sum_{k \leq x m / y^{2}} v^{\Omega(k)} \\
& \ll \frac{\eta x(\log y)^{v-1} \log \log y}{y \log x} \sum_{m \leq y^{2 / 3}} v^{\Omega(m)}(\log 2 m)^{v-1} \sum_{y / m<t_{1} \leq z / m} v^{\Omega\left(t_{1}\right)},
\end{aligned}
$$

uniformly in $1 / 10 \leq v \leq 1$, by Lemma 2.5 and Theorem 01 in [15]. Also,

$$
\begin{aligned}
\sum_{y / m<t_{1} \leq z / m} v^{\Omega\left(t_{1}\right)} \asymp \frac{y}{m} \sum_{y / m<t_{1} \leq z / m} \frac{v^{\Omega\left(t_{1}\right)}}{t_{1}} & \ll \frac{\eta y(\log \log y)^{1-v}}{m}\left(\log \frac{y}{m}\right)^{v-1} \\
& \asymp \frac{\eta y(\log y)^{v-1}(\log \log y)^{1-v}}{m},
\end{aligned}
$$


by (3.21), since $m \leq y^{2 / 3}$. Hence

$$
S_{2}^{\prime} \ll_{s} \frac{\eta^{2} x(\log y)^{2 v-2}(\log \log y)^{2-v}}{\log x} \sum_{m \leq y^{2 / 3}} \frac{v^{\Omega(m)}}{m}(\log 2 m)^{v-1} .
$$

Inequalities (3.19), (3.22) and (3.23) imply that

$$
S^{\prime} \ll_{s} \frac{\eta^{2} x v^{-L(y)}(\log \log y)^{3-2 v}}{\log x} \sum_{m \leq z-y} \frac{v^{\Omega(m)}}{m}(\log 2 m)^{v-1}\left(\log \frac{y}{m}\right)^{2 v-2} .
$$

If we set $v=1 / 2$, by partial summation and the estimate $\sum_{n \leq x} v^{\Omega(n)} \ll x(\log 2 x)^{v-1}$ we find that

$$
\sum_{m \leq z-y} \frac{v^{\Omega(m)}}{m}(\log m)^{v-1}\left(\log \frac{y}{m}\right)^{2 v-2} \ll \frac{\log \log y}{\log y}
$$

and consequently

$$
S^{\prime} \ll_{s} \frac{\eta^{2} x}{\log x}(\log y)^{\log 4-1} 2^{\psi(y) \sqrt{\log \log y}}(\log \log y)^{3} .
$$

Lastly, putting $\psi(y)=\min \left\{\xi,(\log \log y)^{1 / 6}\right\}$ yields that

$$
S^{\prime} \ll_{s} \frac{\eta x}{\log x} \frac{(\log \log y)^{3}}{e^{(1-\log 2) \xi \sqrt{\log \log y}}}=o\left(\frac{\eta x}{\log x}\right) .
$$

Inserting the above estimate and (3.18) into (3.15) gives us that

$$
H\left(x, y, z ; P_{s}\right) \geq f(s) \frac{315 \zeta(3)}{2 \pi^{4}} \frac{\eta x}{\log x}\left(1-o_{s}(1)\right)
$$

which completes the proof of part (d) in the case that $z>y+(\log y)^{-2}$ too.

\section{INTERMEDIATE AND LARGE VALUES OF $\eta$}

To prove Theorem 1.6 we reduce the counting in $H\left(x, y, z ; P_{s}\right)-H\left(x-\Delta, y, z ; P_{s}\right)$ to the estimation of a sum involving $L(a ; \eta)$ as done in [11] for bounding $H(x, y, z)$; then we apply Lemma 2.10. First, we show the following result. Theorem 1.6 will then follow as an easy corollary.

Theorem 4.1. Fix $s \in \mathbb{Z} \backslash\{0\}$ and $B \geq 2$. Let $x \geq x_{0}(s, B)$ and $3 \leq y+1 \leq z$ with $z \leq x^{2 / 3}, \eta \in\left[(\log y)^{-B}, \frac{\log y}{100}\right]$ and $\left.\{y<d \leq z:(d, s)=1\} \neq \emptyset\right\}$. Then for

$$
\frac{x}{(\log x)^{B}} \leq \Delta \leq \frac{x}{2}
$$

we have that

$$
H\left(x, y, z ; P_{s}\right)-H\left(x-\Delta, y, z ; P_{s}\right) \gg_{s, B} \frac{\Delta}{x} \frac{H(x, y, z)}{\log x} .
$$


Proof. Fix $\Delta \in\left(x(\log x)^{-B}, x / 2\right]$ and set $s_{1}=2 /(s, 2)$. Let $y_{0}=y_{0}(s, B)$ be a large positive constant. If $y \leq y_{0}$, then

$$
\begin{aligned}
H\left(x, y, z ; P_{s}\right)-H\left(x-\Delta, y, z ; P_{s}\right) & \geq \max _{\substack{y<d \leq z \\
(d, s)=1}}(\pi(x-s ; d,-s)-\pi(x-\Delta-s ; d,-s)) \\
& \gg_{y_{0}} \frac{\Delta}{\log x} \asymp_{y_{0}} \frac{\Delta}{x} \frac{H(x, y, z)}{\log x},
\end{aligned}
$$

by the Prime Number Theorem for arithmetic progressions [4, p. 123] and our assumption that $\{y<d \leq z:(d, s)=1\} \neq \emptyset$. Suppose now that $y>y_{0}$. Fix an integer $v=v(s) \geq 3$ and set $w=z^{1 / 20 v}$. We will choose $v$ later; till then, all implied constants will be independent of $v$. Consider integers $n=a q b_{1} b_{2} s_{1} \in(x-\Delta, x]$ with

(1) $a \leq w, \mu^{2}(a)=1$ and $(a, 2 s)=1$;

(2) $\log (y / q) \in \mathcal{L}(a ; \eta), P^{-}(q)>w$ and $(q, 2 s)=1$;

(3) $b_{1} \in \mathscr{P}(w, z)$ and $\tau\left(b_{1}\right) \leq v^{2}$;

(4) $P^{-}\left(b_{2}\right)>z$;

(5) $n-s$ is prime.

Condition (2) implies that there exists $d \mid a$ such that $y / d<q \leq z / d$; in particular, we have that $\tau(n, y, z) \geq 1$ and thus $n$ is counted by $H\left(x, y, z ; P_{s}\right)-H\left(x-\Delta, y, z ; P_{s}\right)$. Also, $\Omega(q) \leq \frac{\log z}{\log w}=20 v$ and therefore

$$
\tau\left(q b_{1}\right) \leq 2^{\Omega(q)} \tau\left(b_{1}\right) \leq 2^{20 v} v^{2} .
$$

Since each $n$ has at most $\tau\left(q b_{1}\right) \leq 2^{20 v} v^{2}$ representations of this form, we find that

$$
\begin{aligned}
2^{20 v} v^{2}\left(H\left(x, y, z ; P_{s}\right)-H\left(x-\Delta, y, z ; P_{s}\right)\right) & \geq \sum_{\substack{a \leq w \\
\mu^{2}(a)=1 \\
(a, 2 s)=1}} \sum_{\substack{\log (y / q) \in \mathcal{L}(a ; \eta) \\
P^{-}(q)>w \\
(q, 2 s)=1}} \sum_{\substack{(x-\Delta) / a q s_{1}<b_{1} b_{2} \leq x / a q s_{1} \\
b_{1} \in \mathscr{P}(w, z), P^{-}\left(b_{2}\right)>z \\
\tau\left(b_{1}\right) \leq v^{2} \\
a q b_{1} b_{2} s_{1}-s \text { prime }}} 1 \\
= & \sum_{\substack{a \leq w \\
\mu^{2}(a)=1 \\
(a, 2 s)=1}} \sum_{\substack{\log (y / q) \in \mathcal{L}(a ; \eta) \\
P^{-}(q)>w \\
(q, 2 s)=1}} B_{0}(a, q) .
\end{aligned}
$$

Given $a$ and $q$ as above, put

$$
B(a, q)=\sum_{\substack{(x-\Delta) / a q s_{1}<b \leq x / a q s_{1} \\ P^{-}(b)>w \\ a q b s_{1}-s \text { prime }}} 1 \text { and } R(a, q)=B(a, q)-B_{0}(a, q) .
$$

Given $b$ with $P^{-}(b)>w$, write $b=b_{1} b_{2}$ with $b_{1} \in \mathscr{P}(w, z)$ and $P^{-}\left(b_{2}\right)>z$ and put $F(b)=\tau\left(b_{1}\right)$. Then, for fixed $a$ and $q$ with $(a q, 2 s)=1$, we have that

$$
R(a, q) \leq \frac{1}{v^{2}} \sum_{\substack{(x-\Delta) / a q s_{1}<b \leq x / a q s_{1} \\ P^{-}(b)>w \\ a q b s_{1}-s \text { prime }}} F(b)=\frac{1}{v^{2}} \sum_{\substack{x-\Delta<p+s \leq x \\ p \equiv-s\left(\bmod a q s_{1}\right) \\ P^{-}\left(\frac{p+s}{a q s_{1}}\right)>w}} F\left(\frac{p+s}{a q s_{1}}\right) \ll s \frac{1}{v} \frac{\Delta}{\phi(a q) \log x \log w},
$$


by Lemma 2.5. Inserting the above estimate into (4.1) yields that

$$
\begin{array}{r}
2^{20 v} v^{2}\left(H\left(x, y, z ; P_{s}\right)-H\left(x-\Delta, y, z ; P_{s}\right)\right) \geq \sum_{\substack{a \leq w \\
\mu^{2}(a)=1 \\
(a, 2 s)=1}} \sum_{\substack{\log (y / q) \in \mathcal{L}(a ; \eta) \\
P^{-}(q)>w \\
(q, 2 s)=1}} B(a, q) \\
-O_{s}\left(\frac{1}{v} \frac{\Delta}{\log x \log w} \sum_{\substack{a \leq w \\
\mu^{2}(a)=1 \\
(a, 2 s)=1}} \sum_{\substack{P^{-}(q)>w \\
(q, 2 s)=1}} \frac{1}{\phi(a q)}\right) .
\end{array}
$$

Next, we need to approximate the characteristic function of integers $n$ with $P^{-}(n)>w$ with a 'smoother' function, the reason being that the error term $\pi(x ; r q, a)-\operatorname{li}(x) / \phi(r q)$ in Lemma 2.7 is weighted with the smooth function 1 as $q$ runs through $[1, Q] \cap \mathbb{N}$. To do this we appeal to Lemma 2.9(a) with $Z=w, D=z^{1 / 20}$ and $\kappa=2$. Then

$$
\begin{aligned}
2^{20 v} v^{2}\left(H\left(x, y, z ; P_{s}\right)-H\left(x-\Delta, y, z ; P_{s}\right)\right) & \sum_{\substack{a \leq w \\
\mu^{2}(a)=1,(a, 2 s)=1}} \sum_{\substack{\log (y / q) \in \mathcal{L}(a ; \eta) \\
(q, 2 s)=1}}\left(\lambda^{-} * 1\right)(q) B(a, q)-O_{s}\left(\mathscr{R}_{1}\right) \\
\geq & \sum_{\substack{a \leq w \\
\mu^{2}(a)=1,(a, 2 s)=1}} \sum_{\substack{\log (y / q) \in \mathcal{L}(a ; \eta) \\
(q, 2 s)=1}}\left(\lambda^{+} * 1\right)(q) B(a, q)-O_{s}\left(\mathscr{R}_{1}+\mathscr{R}_{2}\right),
\end{aligned}
$$

where

$$
\mathscr{R}_{1}:=\frac{1}{v} \frac{\Delta}{\log x \log w} \sum_{\substack{a \leq w \\ \mu^{2}(a)=1,(a, 2 s)=1}} \sum_{\substack{\log (y / q) \in \mathcal{L}(a ; \eta) \\(q, 2 s)=1}} \frac{\left(\lambda^{+} * 1\right)(q)}{\phi(a q)}
$$

and

$$
\mathscr{R}_{2}:=\sum_{\substack{a \leq w \\ \mu^{2}(a)=1,(a, 2 s)=1}} \sum_{\substack{\log (y / q) \in \mathcal{L}(a ; \eta) \\(q, 2 s)=1}}\left(\left(\lambda^{+} * 1\right)(q)-\left(\lambda^{-} * 1\right)(q)\right) B(a, q) .
$$

We now bound $\mathscr{R}_{2}$ from above. For fixed $a$ and $q$ with $(a q, 2 s)=1$ we have

$$
B(a, q) \ll_{s} \frac{\Delta}{\phi(a q) \log x \log w}
$$

by the arithmetic form of the large sieve [21] or Lemma 2.5. Since $\lambda^{+} * 1-\lambda^{-} * 1$ is always non-negative, we get that

$$
\mathscr{R}_{2} \ll \frac{\Delta}{\log x \log w} \sum_{\substack{a \leq w \\ \mu^{2}(a)=1,(a, 2 s)=1}} \sum_{\substack{\log (y / q) \in \mathcal{L}(a ; \eta) \\(q, 2 s)=1}} \frac{\left(\lambda^{+} * 1\right)(q)-\left(\lambda^{-} * 1\right)(q)}{\phi(a q)} .
$$


Fix $a \leq w$ with $(a, 2 s)=1$ and let $\left\{I_{r}\right\}_{r=1}^{R}$ be the collection of the intervals $[\log d-\eta, \log d)$ with $d \mid a$. Then for $I=[\log d-\eta, \log d)$ in this collection Lemmas 2.8 and 2.9(a) imply that

$$
\begin{aligned}
\sum_{\substack{\log (y / q) \in 3 I \\
(q, 2 s)=1}} & \frac{\left(\lambda^{+} * 1\right)(q)-\left(\lambda^{-} * 1\right)(q)}{\phi(a q)} \\
= & \sum_{\substack{c \leq z^{1 / 20} \\
(c, 2 s)=1}}\left(\lambda^{+}(c)-\lambda^{-}(c)\right) \sum_{\substack{e^{-\eta} y / c d<m \leq e^{2 \eta} y / c d \\
(m, 2 s)=1}} \frac{1}{\phi(a c m)} \\
= & \frac{315 \zeta(3)}{2 \pi^{4}} \frac{g(2 a s) \phi(2 s)}{2|s| \phi(a)} \sum_{\substack{c \leq z^{1 / 20} \\
(c, 2 s)=1}} \frac{\lambda^{+}(c)-\lambda^{-}(c)}{c} \frac{g(a c)}{g(a)} \frac{c \phi(a)}{\phi(a c)}\left(3 \eta+O_{s}\left(y^{-2 / 3}\right)\right) \\
\ll s & \frac{\eta}{e^{v} \phi(a)} \prod_{\substack{p \leq w \\
p \nmid 2 s, p \mid a}}\left(1-\frac{1}{p}\right) \prod_{\substack{p \leq w \\
p \nmid 2 s a}}\left(1-\frac{g(p)}{p-1}\right)+\frac{1}{\phi(a) \sqrt{y}} \asymp s \frac{1}{e^{v}} \frac{\eta}{\phi(a) \log w},
\end{aligned}
$$

provided that $y_{0}$ is large enough, since $g(p) p /(p-1) \leq \min \{p-1,2\}$ for $p \geq 3, g(p)=$ $1+O\left(p^{-2}\right)$ and $g(a) \asymp 1$. By Lemma 2.12, there exists a sub-collection $\left\{I_{r_{t}}\right\}_{t=1}^{T}$ of mutually disjoint intervals so that

$$
\bigcup_{t=1}^{T} 3 I_{r_{t}} \supset \mathcal{L}(a ; \eta)
$$

Consequently

$$
\begin{aligned}
\sum_{\substack{\log (y / q) \in \mathcal{L}(a ; \eta) \\
(q, 2 s)=1}} \frac{\left(\lambda^{+} * 1\right)(q)-\left(\lambda^{-} * 1\right)(q)}{\phi(a q)} & \leq \sum_{t=1}^{T} \sum_{\substack{\log (y / q) \in 3 I_{r_{t}} \\
(q, 2 s)=1}} \frac{\left(\lambda^{+} * 1\right)(q)-\left(\lambda^{-} * 1\right)(q)}{\phi(a q)} \\
& \ll_{s} \frac{1}{e^{v}} \frac{T \eta}{\phi(a) \log w} \\
& =\frac{1}{e^{v}} \frac{1}{\phi(a) \log w} \operatorname{meas}\left(\bigcup_{t=1}^{T} I_{r_{t}}\right) \\
& \leq \frac{1}{e^{v}} \frac{L(a ; \eta)}{\phi(a) \log w},
\end{aligned}
$$

since $\lambda^{+} * 1-\lambda^{-} * 1$ is always non-negative. By the above inequality and (4.4) we get that

$$
\mathscr{R}_{2} \ll_{s} \frac{1}{e^{v}} \frac{\Delta}{\log x \log ^{2} w} \sum_{\substack{a \leq w \\ \mu^{2}(a)=1,(a, 2 s)=1}} \frac{L(a ; \eta)}{\phi(a)} .
$$

We now bound from the below the sum

$$
\mathscr{S}:=\sum_{\substack{a \leq w \\ \mu^{2}(a)=1,(a, 2 s)=1}} \sum_{\substack{\log (y / q) \in \mathcal{L}(a ; \eta) \\(q, 2 s)=1}}\left(\lambda^{+} * 1\right)(q) B(a, q) .
$$


We fix $a$ and $q$ with $(a q, 2 s)=1$ and seek a lower bound on $B(a, q)$. By Lemma 2.9(b) applied with $Z=w$ and $D=w^{3}$, there exists a sequence $\{\rho(d)\}_{d \leq w^{3}}$ such that $\rho * 1$ is bounded above by the characteristic function of integers $b$ with $P^{-}(b)>w$. So, if we put

$$
E(x ; k, a)=\pi(x-s ; k, a)-\pi(x-\Delta-s ; k, a)-\frac{\operatorname{li}(x-s)-\operatorname{li}(x-\Delta-s)}{\phi(k)},
$$

then Lemma 2.9(b) and the fact that $2 \mid s_{1} s$ imply that

$$
\begin{aligned}
B(a, q) & =\sum_{\substack{x-\Delta<p+s \leq x \\
p \equiv-s\left(\bmod a q s_{1}\right) \\
P^{-}\left((p+s) / a q s_{1}\right)>w}} 1 \geq \sum_{\substack{x-\Delta<p+s \leq x \\
p \equiv-s\left(\bmod a q s_{1}\right) \\
p \nmid s}}(\rho * 1)\left(\frac{p+s}{a q s_{1}}\right) \\
& =\sum_{\substack{m \leq w^{3} \\
(m, s)=1}} \rho(m)\left(\pi\left(x-s ; a q s_{1} m,-s\right)-\pi\left(x-s-\Delta ; a q s_{1} m,-s\right)\right)+O_{s}(1) \\
& =(\operatorname{li}(x-s)-\operatorname{li}(x-s-\Delta)) \sum_{\substack{m \leq w^{3} \\
(m, s)=1}} \frac{\rho(m)}{\phi\left(a q s_{1} m\right)}+O_{s}(1)+\mathscr{R}_{a q s_{1}}^{\prime} \\
& \geq C_{s} \frac{\Delta}{\phi(a q) \log x \log w}+\mathscr{R}_{a q s_{1}}^{\prime}
\end{aligned}
$$

for some positive constant $C_{s}$, where

$$
\mathscr{R}_{a q s_{1}}^{\prime}=\sum_{\substack{m \leq w^{3} \\(m, s)=1}} \rho(m) E\left(x ; a q s_{1} m,-s\right) .
$$

Since $\lambda^{+} * 1$ is always non-negative, we deduce that

$$
\mathscr{S} \geq C_{s} \frac{\Delta}{\log x \log w} \sum_{\substack{a \leq w \\ \mu^{2}(a)=1,(a, 2 s)=1}} \sum_{\substack{\log (y / q) \in \mathcal{L}(a ; \eta) \\(q, 2 s)=1}} \frac{\left(\lambda^{+} * 1\right)(q)}{\phi(a q)}+\mathscr{R}^{\prime},
$$

where

$$
\mathscr{R}^{\prime}=\sum_{\substack{a \leq w \\ \mu^{2}(a)=1,(a, 2 s)=1}} \sum_{\substack{\log (y / q) \in \mathcal{L}(a ; \eta) \\(q, 2 s)=1}}\left(\lambda^{+} * 1\right)(q) \mathscr{R}_{a q s_{1}}^{\prime} .
$$

Combining (4.3), (4.6) and (4.7) we get that

$$
\begin{aligned}
2^{20 v} v^{2}( & \left.H\left(x, y, z ; P_{s}\right)-H\left(x-\Delta, y, z ; P_{s}\right)\right) \\
\geq & \frac{C_{s}}{2} \frac{\Delta}{\log x \log w} \sum_{\substack{a \leq w \\
\mu^{2}(a)=1,(a, 2 s)=1}} \sum_{\substack{\log (y / q) \in \mathcal{L}(a ; \eta) \\
(q, 2 s)=1}} \frac{\left(\lambda^{+} * 1\right)(q)}{\phi(a q)} \\
& -O_{s}\left(\left|\mathscr{R}^{\prime}\right|+\frac{1}{e^{v}} \frac{\Delta}{\log x \log ^{2} w} \sum_{\substack{a \leq w \\
\mu^{2}(a)=1,(a, 2 s)=1}} \frac{L(a ; \eta)}{\phi(a)}\right),
\end{aligned}
$$


provided that $v$ is large enough. Fix now $a \leq w$ with $(a, 2 s)=1$ and look at the sum over $q$ on the right hand side of (4.8). Let $\left\{I_{r}\right\}_{r=1}^{R}$ be the collection of the intervals $[\log d-\eta, \log d)$ with $d \mid a$. Then, using a similar argument with the one leading to (4.5), we find that for $I$ in this collection

$$
\sum_{\substack{\log (y / q) \in I \\(q, 2 s)=1}} \frac{\left(\lambda^{+} * 1\right)(q)}{\phi(a q)} \gg_{s} \frac{\eta}{\phi(a) \log w}
$$

provided that $y_{0}$ and $v$ are large enough. Moreover, by Lemma 2.12, there exists a subcollection $\left\{I_{r_{t}}\right\}_{t=1}^{T}$ of mutually disjoint intervals so that

$$
\eta T=\operatorname{Vol}\left(\bigcup_{t=1}^{T} I_{r_{t}}\right) \geq \frac{1}{3} \operatorname{Vol}\left(\bigcup_{r=1}^{R} I_{r}\right)=\frac{L(a ; \eta)}{3} .
$$

Hence

$$
\sum_{\substack{\log (y / q) \in \mathcal{L}(a ; \eta) \\(q, 2 s)=1}} \frac{\left(\lambda^{+} * 1\right)(q)}{\phi(a q)} \geq \sum_{t=1}^{T} \sum_{\substack{\log (y / q) \in I_{r_{t}} \\(q, 2 s)=1}} \frac{\left(\lambda^{+} * 1\right)(q)}{\phi(a q)} \gg_{s} \frac{\eta T}{\phi(a) \log w} \gg \frac{L(a ; \eta)}{\phi(a) \log w}
$$

where we used the fact that $\lambda^{+} * 1$ is non-negative. Inserting this inequality into (4.8) and choosing a large enough $v$ we conclude that

$$
H\left(x, y, z ; P_{s}\right)-H\left(x-\Delta, y, z ; P_{s}\right) \geq M_{s} \frac{\Delta}{\log x \log ^{2} y} \sum_{\substack{a \leq w \\ \mu^{2}(a)=1,(a, 2 s)=1}} \frac{L(a ; \eta)}{\phi(a)}-O_{s}\left(\left|\mathscr{R}^{\prime}\right|\right)
$$

for some positive constant $M_{s}$. Furthermore, note that if $a$ is squarefree, we may uniquely write $a=d b$, where $d \mid 2 s, \mu^{2}(d)=\mu^{2}(b)=1$ and $(b, 2 s)=1$, in which case $L(a ; \eta) \leq$ $\tau(d) L(b ; \eta)$, by inequality (2.12). Thus

$$
\sum_{\substack{a \leq w \\ \mu^{2}(a)=1}} \frac{L(a ; \eta)}{\phi(a)} \leq \sum_{d \mid 2 s, \mu^{2}(d)=1} \frac{\tau(d)}{\phi(d)} \sum_{\substack{b \leq w / d \\ \mu^{2}(b)=1 \\(b, 2 s)=1}} \frac{L(b ; \eta)}{\phi(b)} \leq\left(\sum_{d \mid s} \frac{\tau(d)}{\phi(d)}\right) \sum_{\substack{b \leq w \\ \mu^{2}(b)=1 \\(b, 2 s)=1}} \frac{L(b ; \eta)}{\phi(b)}
$$

which, combined with (4.9), Lemma 2.10 and the trivial inequality $\phi(a) \leq a$, implies that

$$
H\left(x, y, z ; P_{s}\right)-H\left(x-\Delta, y, z ; P_{s}\right) \geq M_{s}^{\prime} \frac{\Delta}{x} \frac{H(x, y, z)}{\log x}-O_{s}\left(\left|\mathscr{R}^{\prime}\right|\right)
$$

for some positive constant $M_{s}^{\prime}$. In addition, observe that

$$
H(x, y, z) \gg \frac{x}{(\log y)^{B}},
$$

by Theorem 1.1 and our assumption that $\eta \geq(\log y)^{-B}$. Hence

$$
H\left(x, y, z ; P_{s}\right)-H\left(x-\Delta, y, z ; P_{s}\right) \gg_{s} \frac{\Delta}{x} \frac{H(x, y, z)}{\log x}\left(1-O_{s}\left(\frac{(\log x)(\log y)^{B}\left|\mathscr{R}^{\prime}\right|}{\Delta}\right)\right) .
$$


So it suffices to show that

$$
\left|\mathscr{R}^{\prime}\right| \ll_{s} \frac{\Delta}{(\log x)(\log y)^{B+1}} .
$$

For every $a \in \mathbb{N}$ there is a unique set $D_{a}$ of pairs $\left(d, d^{\prime}\right)$ with $d \leq d^{\prime}, d \mid a$ and $d^{\prime} \mid a$ such that

$$
\mathcal{L}(a ; \eta)=\bigcup_{\left(d, d^{\prime}\right) \in D_{a}}\left[\log d-\eta, \log d^{\prime}\right)
$$

and the intervals $\left[\log d-\eta, \log d^{\prime}\right)$ for $\left(d, d^{\prime}\right) \in D_{a}$ are mutually disjoint. With this notation we have that

$$
\begin{aligned}
& \left|\mathscr{R}^{\prime}\right|=\left|\sum_{a} \sum_{m} \rho(m) \sum_{\left(d, d^{\prime}\right) \in D_{a}} \sum_{\begin{array}{r}
y / d^{\prime}<q \leq z / d \\
(q, 2 s)=1
\end{array}}\left(\lambda^{+} * 1\right)(q) E\left(x ; a m s_{1} q,-s\right)\right| \\
& =\left|\sum_{a} \sum_{m} \rho(m) \sum_{\left(d, d^{\prime}\right) \in D_{a}} \sum_{c} \lambda^{+}(c) \sum_{\begin{array}{c}
y / c d^{\prime}<g \leq z / c d \\
(g, 2 s)=1
\end{array}} E\left(x ; a m s_{1} c g,-s\right)\right| \\
& \leq \sum_{\substack{a \leq w \\
(a, 2 s)=1}} \sum_{\substack{m \leq w^{3} \\
(m, s)=1}} \sum_{\substack{c \leq z^{1 / 20} \\
(c, 2 s)=1}}\left|\sum_{\left(d, d^{\prime}\right) \in D_{a}} E\left(x ; a m s_{1} c g,-s\right)\right| .
\end{aligned}
$$

So writing the inner sum as a difference of two sums we obtain that

$$
\begin{aligned}
\left|\mathscr{R}^{\prime}\right| & \leq 2 \sup _{y \leq t \leq z}\left\{\sum_{\substack{a \leq w \\
(a, 2 s)=1}} \sum_{\substack{m \leq w^{3} \\
(m, s)=1}} \sum_{\substack{c \leq z^{1 / 20} \\
(c, 2 s)=1}} \sum_{f \mid a m s_{1} c}\left|\sum_{\substack{g \leq t / f \\
(g, 2 s)=1}} E\left(x ; a m s_{1} c g,-s\right)\right|\right\} \\
& \leq 2 \sup _{y \leq t \leq z}\left\{\sum_{\substack{r \leq 2 z^{7 / 60} \\
(r, s)=1}} \tau_{3}(r) \sum_{f \mid r}\left|\sum_{\substack{g \leq t / f \\
(g, 2 s)=1}} E(x ; r g,-s)\right|\right\} \\
& \leq 4 \sup _{y \leq t \leq z}\left\{\sum_{\substack{r \leq z^{1 / 8} \\
(r, s)=1}} \tau_{3}(r) \sum_{f \mid r}\left|\sum_{\substack{g \leq t / f \\
(g, s)=1}} E(x ; r g,-s)\right|\right\},
\end{aligned}
$$

since $w^{4} z^{1 / 20} \leq z^{7 / 60} \leq z^{1 / 8} / 4$ for all $v \geq 3$. Put $\mu=1+(\log y)^{-B-7}$ and cover the interval $\left[1, z^{1 / 8}\right]$ by intervals of the form $\left[\mu^{n}, \mu^{n+1}\right)$ for $n=0,1, \ldots, N$. We may take $N \ll(\log y)^{B+8}$. Since $|E(x ; k,-s)| \ll \frac{\Delta}{\phi(k) \log x}$ for $k \leq z^{9 / 8} \leq x^{3 / 4}$ with $(k, s)=1$ by Lemma 2.4 , we have 
that

$$
\begin{aligned}
& \sum_{\substack{r \leq z^{1 / 8} \\
(r, s)=1}} \tau_{3}(r) \sum_{n=0}^{N} \sum_{\substack{f \mid r \\
\mu^{n} \leq f<\mu^{n+1}}}\left|\sum_{\substack{g \leq t / f \\
(g, s)=1}} E(x ; r g,-s)-\sum_{\substack{g \leq t / \mu^{n} \\
(g, s)=1}} E(x ; r g,-s)\right| \\
& \ll \sum_{\substack{r \leq z^{1 / 8} \\
(r, s)=1}} \tau_{3}(r) \sum_{n=0}^{N} \sum_{\substack{f \mid r \\
\mu^{n} \leq f<\mu^{n+1}}} \sum_{\substack{t / \mu^{n+1}<g \leq t / \mu^{n} \\
\phi(r g) \log x}} \frac{\Delta}{\phi} \\
& \ll \frac{\Delta \log \mu}{\log x} \sum_{r \leq z^{1 / 8}} \frac{\tau_{3}(r)}{\phi(r)} \sum_{f \mid r} 1 \ll \frac{\Delta}{(\log x)(\log y)^{B+1}}
\end{aligned}
$$

for all $t \in[y, z]$, by Lemma 2.8 , which is admissible. Combining the above estimate with (4.10) we find that

$$
\left|\mathscr{R}^{\prime}\right| \ll \sup _{y \leq t \leq z}\left\{\sum_{n=0}^{N} \sum_{\substack{r \leq z^{1 / 8} \\(r, s)=1}} \tau_{3}(r) \tau(r)\left|\sum_{\substack{g \leq t / \mu^{n} \\(g, s)=1}} E(x ; r g,-s)\right|\right\}+\frac{\Delta}{(\log x)(\log y)^{B+1}} .
$$

Finally, since

$$
\frac{x}{2} \leq x-\Delta \leq x \quad \text { and } \quad \Delta \geq \frac{x}{(\log x)^{B}}
$$

Lemma 2.7 applied with $A=5 B+56$ in combination with the Cauchy-Schwarz inequality yields that

$$
\begin{aligned}
& \sum_{\substack{r \leq z^{1 / 8} \\
(r, s)=1}} \tau_{3}(r) \tau(r)\left|\sum_{\substack{g \leq t / \mu^{n} \\
(g, s)=1}} E(x ; r g,-s)\right| \\
& \ll\left(\frac{\Delta}{\log x} \sum_{r \leq z^{1 / 8}} \sum_{g \leq t / \mu^{n}} \frac{\left(\tau_{3}(r) \tau(r)\right)^{2}}{\phi(r g)}\right)^{1 / 2}\left(\sum_{\substack{r \leq z^{1 / 8} \\
(r, s)=1}}\left|\sum_{\substack{g \leq t / \mu^{n} \\
(g, s)=1}} E(x ; r g,-s)\right|\right)^{1 / 2} \\
& \ll_{s} \sqrt{\Delta}(\log x)^{18} \frac{\sqrt{x}}{(\log x)^{5 B / 2+28}} \leq \frac{\Delta}{(\log x)^{2 B+10}}
\end{aligned}
$$

for all $t \in[y, z]$ and all $n \in\{0,1, \ldots, N\}$, since $z^{1 / 8} \leq x^{1 / 12}$ and $z^{9 / 8} \leq x^{3 / 4}$. Plugging this estimate into (4.11) gives us that

$$
\left|\mathscr{R}^{\prime}\right| \ll_{s} N \frac{\Delta}{(\log x)^{2 B+10}}+\frac{\Delta}{(\log x)(\log y)^{B+1}} \ll \frac{\Delta}{(\log x)(\log y)^{B+1}},
$$

which is admissible.

We are now in position to complete the proof of Theorem 1.6.

Proof of Theorem [1.6. Fix $\Delta \in\left(x(\log x)^{-B}, x\right]$ and set $\Delta_{1}=\min \{\Delta, x / 2\}$. If $\eta \leq \frac{\log y}{100}$, then the theorem follows immediately by Theorem 4.1 and the trivial inequality

$$
H\left(x, y, z ; P_{s}\right)-H\left(x-\Delta, y, z ; P_{s}\right) \geq H\left(x, y, z ; P_{s}\right)-H\left(x-\Delta_{1}, y, z ; P_{s}\right) .
$$


On the other hand, if $\eta \geq \frac{\log y}{100}$, then

$$
\begin{aligned}
& H\left(x, y, z ; P_{s}\right)-H\left(x-\Delta, y, z ; P_{s}\right) \geq H\left(x, y, y^{101 / 100} ; P_{s}\right)-H\left(x-\Delta, y, y^{101 / 100} ; P_{s}\right) \\
& \gg_{s} \frac{\Delta}{x} \frac{H\left(x, y, y^{101 / 100}\right)}{\log x} \asymp \frac{\Delta}{x} \frac{H(x, y, z)}{\log x},
\end{aligned}
$$

by Theorem 1.1. In any case, Theorem 1.6 holds.

Using Theorems 1.3 and 1.6 together with the fact that if $d \mid n$, then $(n / d) \mid d$ as well, we show Theorem 1.7 .

Proof of Theorem 1.7. We may assume that $y>\sqrt{x}$; else the result follows from Theorems 1.3 and 1.6 with $\Delta=x$. For future reference, note the trivial inequality

$$
H\left(x, y, z ; P_{s}\right) \geq \pi(z-s)-\pi(y-s) \asymp_{s, B} \frac{z-y}{\log z} \geq \frac{\eta y}{\log x},
$$

by the Prime Number Theorem. First, suppose that $\eta \leq \log ^{-2}(5 x / z)$. For $q \in \mathbb{N}$ set

$$
A_{q}=\{p+s \in(q y, q z]: p \equiv-s \quad(\bmod q)\} .
$$

If the shifted prime $p+s \leq x$ has a divisor $d \in(y, z]$, then writing $p+s=d q$ we have that $q \leq x / y$ and $p+s \in A_{q}$. So, by Lemma 2.4, we find that

$$
\begin{aligned}
H\left(x, y, z ; P_{s}\right) \leq \sum_{\substack{q \leq x / y \\
(q, s)=1}}\left|A_{q}\right|+O_{s}(1) \ll_{s} \sum_{\substack{q \leq x / y \\
(q, s)=1}} \frac{q(z-y)}{\phi(q) \log (z-y)} & \asymp B \frac{\eta y}{\log x} \sum_{\substack{q \leq x / y \\
(q, s)=1}} \frac{q}{\phi(q)} \\
& \ll \frac{\eta x}{\log x} \asymp \frac{H(x, y, z)}{\log x},
\end{aligned}
$$

by Theorem 1.1. This proves the upper bound in Theorem 1.7 when $\eta \leq \log ^{-2}(5 x / z)$. In order to show the lower bound when $\eta \leq \log ^{-2}(5 x / z)$ it suffices to consider the case $z>x^{2 / 3}$, since if $z \leq x^{2 / 3}$, then we immediately obtain the result by Theorem 4.1. If $x / z \leq 2|s|+2$, then $y \asymp_{s} x$ and thus

$$
H\left(x, y, z ; P_{s}\right) \gg_{s, B} \frac{\eta x}{\log x},
$$

by (4.12). Combining this with Theorem 1.1 we complete the proof in this case. So assume that $x / z \geq 2|s|+2$, in which case

$$
\{x / 2 z<q \leq x / z:(q, s)=1\} \neq \emptyset .
$$

It is easy to see that

$$
H\left(x, y, z ; P_{s}\right) \geq\left|\bigcup_{\substack{x / 2 z<q \leq x / z \\(q, s)=1}} A_{q}\right| .
$$

If we set

$$
T(p)=\left|\left\{x / 2 z<q \leq x / z:(q, s)=1, p+s \in A_{q}\right\}\right|,
$$


then the Cauchy-Schwarz inequality and (4.14) yield that

$$
\left(\sum_{p+s \leq x} T(p)\right)^{2} \leq H\left(x, y, z ; P_{s}\right) \sum_{p+s \leq x} T^{2}(p) .
$$

First, we estimate $\sum_{p+s \leq x} T(p)$. Let $C=C(B)>0$ be a constant so that

$$
\sum_{\substack{q \leq Q \\(q, s)=1}} \pi(X ; q,-s)=\operatorname{li}(X) \sum_{\substack{q \leq Q \\(q, s)=1}} \frac{1}{\phi(q)}+O_{s, B}\left(\frac{X}{(\log X)^{B+2}}\right)
$$

for all $X \geq 2$ and all $Q \leq X(\log X)^{-C}$. Such a constant exists by Lemma 2.7. If $x / z \leq$ $(\log x)^{C+1}$, then the Siegel-Walfisz theorem [4, p. 133] and Lemma 2.8 imply that

$$
\begin{aligned}
\sum_{p+s \leq x} T(p) & =\sum_{\substack{x / 2 z<q \leq x / z \\
(q, s)=1}}(\pi(q z-s ; q,-s)-\pi(q y-s ; q,-s)) \\
& \gg_{s, B} \sum_{\substack{x / 2 z<q \leq x / z \\
(q, s)=1}} \frac{q(z-y)}{\phi(q) \log x} \asymp \frac{\eta x}{\log x} \sum_{\substack{x / 2 z<q \leq x / z \\
(q, s)=1}} \frac{1}{\phi(q)} \\
& \asymp_{s} \frac{\eta x}{\log x} .
\end{aligned}
$$

On the other hand, if $x / z \geq(\log x)^{C+1}$, then (4.16) and Lemma 2.8 yield that

$$
\begin{aligned}
\sum_{p+s \leq x} T(p) & \geq \sum_{x / 2<p+s \leq 2 x / 3} \sum_{\substack{p+s \leq q<\frac{p+s}{y} \\
(q, s)=1, q \mid(p+s)}} 1 \\
& =\sum_{\substack{y<d \leq z \\
(d, s)=1}}(\pi(2 x / 3-s ; d,-s)-\pi(x / 2-s ; d,-s))+O_{s}(1) \\
& =(\operatorname{li}(2 x / 3-s)-\operatorname{li}(x / 2-s)) \sum_{\substack{y<d \leq z \\
(d, s)=1}} \frac{1}{\phi(d)}+O_{s, B}\left(\frac{x}{(\log x)^{B+2}}\right) \\
& \asymp_{s, B} \frac{\eta x}{\log x},
\end{aligned}
$$

since $\eta \leq \log ^{-2} 5 \leq \log (3 / 2)$. Also,

$$
\sum_{p+s \leq x} T(p)=\sum_{\substack{x / 2 z<q \leq x / z \\(q, s)=1}}\left|A_{q}\right| \ll_{s, B} \frac{\eta x}{\log x}
$$

by (4.13). Combining inequalities (4.17), (4.18) and (4.19) we deduce that

$$
\sum_{p+s \leq x} T(p) \asymp_{s, B} \frac{\eta x}{\log x},
$$


uniformly in $\eta \leq \log ^{-2}(5 x / z)$ and $x / z \geq 2|s|+2$. We now bound from above the sum

$$
\sum_{p+s \leq x} T^{2}(p)=\sum_{p+s \leq x} T(p)+\sum_{p+s \leq x} T(p)(T(p)-1) .
$$

We have that

$$
\begin{aligned}
\sum_{p+s \leq x} T(p)(T(p)-1) & =\sum_{p} \sum_{\substack{x / 2 z<q_{1} \leq x / z \\
\frac{p+s}{z} \leq q_{1}<\frac{p+s}{y} \\
q_{1} \mid(p+s),\left(q_{1}, s\right)=1}} \sum_{\substack{x / 2 z<q_{2} \leq x / z \\
\frac{p+s}{z} \leq q_{2}<\frac{p+s}{y} \\
q_{2} \mid(p+s),\left(q_{2}, s\right)=1 \\
q_{2} \neq q_{1}}} 1 \\
& =2 \sum_{\substack{\frac{x}{2 z}<q_{1}<q_{2} \leq \frac{x}{z} \\
\left(q_{1} q_{2}, s\right)=1}} \sum_{\substack{p \equiv-s\left(\bmod \left[q_{1}, q_{2}\right]\right) \\
q_{2}}<p+s \leq q_{1} z} 1 .
\end{aligned}
$$

Note that we must have $q_{2}<e^{\eta} q_{1}$; otherwise, the corresponding summand on the right hand side of (4.22) is trivially zero. Lemma 2.4 and the trivial estimate $\pi(x+h ; q, a)-\pi(x ; q, a) \leq$ $h / q+1$ imply

$$
\sum_{\substack{p \equiv-s\left(\bmod \left[q_{1}, q_{2}\right]\right) \\ q_{2} y<p+s \leq q_{1} z}} 1 \ll_{s} \frac{q_{1} z-q_{2} y}{\phi\left(\left[q_{1}, q_{2}\right]\right) \log \left(3+\left(q_{1} z-q_{2} y\right) /\left[q_{1}, q_{2}\right]\right)}+1 .
$$

Set $m=\left(q_{1}, q_{2}\right)$ and $q_{i}=m t_{i}, i=1,2$, in the right hand side of (4.22). Then we will have $m \leq x / 2 z$ and $t_{1}<t_{2}<e^{\eta} t_{1}$. With this notation (4.22) and (4.23) yield that

$$
\begin{aligned}
\sum_{p+s \leq x} T(p)(T(p)-1) & \ll s \log \log (x / y) \sum_{m \leq \frac{x}{2 z}} \sum_{\frac{x}{2 m z}<t_{1} \leq \frac{x}{m z}} \sum_{t_{1}<t_{2}<e^{\eta} t_{1}} \frac{z / t_{2}-y / t_{1}}{\log \left(3+z / t_{2}-y / t_{1}\right)} \\
+ & \frac{x}{z} \log (x / z)+\eta\left(\frac{x}{z}\right)^{2}
\end{aligned}
$$

Fix $m$ and $t_{1}$. Recall that we have assumed that $z>x^{2 / 3}$ and $(\log x)^{-B} \ll \eta \leq(\log (5 x / z))^{-2}$. So $\log \frac{z-y}{t_{1}} \gg_{B} \log x$ and consequently

$$
\begin{aligned}
\sum_{t_{1}<t_{2}<e^{\eta} t_{1}} \frac{z / t_{2}-y / t_{1}}{\log \left(3+z / t_{2}-y / t_{1}\right)} & \leq \int_{t_{1}}^{e^{\eta} t_{1}} \frac{z / u-y / t_{1}}{\log \left(3+z / u-y / t_{1}\right)} d u \\
& =\int_{0}^{(z-y) / t_{1}} \frac{w}{\log (w+3)} \frac{z}{\left(w+y / t_{1}\right)^{2}} d w \\
& \asymp_{B} \frac{\eta^{2} y}{\log x}
\end{aligned}
$$

which, combined with (4.20), (4.21) and (4.24), yields that

$$
\sum_{p+s \leq x} T^{2}(p) \ll_{s, B} \frac{\eta x}{\log x}+\frac{\eta^{2} x}{\log x} \log (x / y) \log \log (x / y) \ll \frac{\eta x}{\log x} .
$$


Plugging the above estimate and (4.20) into (4.15) gives us that

$$
H\left(x, y, z ; P_{s}\right) \gg_{s, B} \frac{\eta x}{\log x} \asymp \frac{H(x, y, z)}{\log x},
$$

by Theorem 1.1. This completes the proof of the theorem in the case when $\eta \leq \log ^{-2}(5 x / z)$. Assume now that $\eta \geq \log ^{-2}(5 x / z)$. Fix a large positive constant $y_{0}=y_{0}(s, B)$. If $x / z \leq y_{0}$, then $\eta \geq \log ^{-2}\left(5 y_{0}\right)$. Hence (4.12) implies that

$$
H\left(x, y, z ; P_{s}\right) \gg_{s, B} \frac{z-y}{\log y} \gg_{y_{0}} \frac{z}{\log y} \gg_{y_{0}} \frac{x}{\log x} .
$$

Combining the above inequality with the trivial estimate $H\left(x, y, z ; P_{s}\right) \leq \pi(x-s)$ and Theorem 1.1 we deduce that

$$
H\left(x, y, z ; P_{s}\right) \asymp_{y_{0}} \frac{x}{\log x} \asymp_{y_{0}} \frac{H(x, y, z)}{\log x},
$$

which shows the desired result in this case. So suppose that $x / z>y_{0}$. We proceed as in the proof of Theorem 1 (iv) in [11]. Partition $\left(\frac{x}{\log ^{2}(x / z)}, x\right]$ into intervals $\left(x_{1}, x_{2}\right]$ with

$$
\frac{x_{2}}{\log ^{3}(x / z)} \leq x_{2}-x_{1} \leq \frac{2 x_{2}}{\log ^{3}(x / z)} \text {. }
$$

Observe that if $p+s \in\left(x_{1}, x_{2}\right]$, then

$$
\tau\left(p+s, \frac{x_{2}}{z}, \frac{x_{1}}{y}\right) \geq 1 \Rightarrow \tau(p+s, y, z) \geq 1 \Rightarrow \tau\left(p+s, \frac{x_{1}}{z}, \frac{x_{2}}{y}\right) \geq 1 .
$$

So

$$
H\left(x, y, z ; P_{s}\right) \leq \sum_{x_{1}, x_{2}}\left\{H\left(x_{2}, \frac{x_{1}}{z}, \frac{x_{2}}{y} ; P_{s}\right)-H\left(x_{1}, \frac{x_{1}}{z}, \frac{x_{2}}{y} ; P_{s}\right)\right\}+O_{s}\left(\frac{x}{\log x \log ^{2}(x / z)}\right) .
$$

Fix such an interval $\left(x_{1}, x_{2}\right]$. Then

$$
\log \left(\frac{x_{1}}{z}\right) \asymp \log \left(\frac{x}{z}\right), \quad x_{2}-x_{1} \geq \frac{x_{2}}{\log ^{4}\left(x_{2} / y\right)}, \quad \log \left(\frac{x_{2} / y}{x_{1} / z}\right) \asymp \eta, \quad \frac{x_{1}}{z} \leq \sqrt{x_{2}},
$$

provided that $y_{0}$ is large enough. Therefore Theorems 1.1 and 1.3 and Lemma 2.11 imply that

$$
\begin{aligned}
H\left(x_{2}, \frac{x_{1}}{z}, \frac{x_{2}}{y} ; P_{s}\right)-H\left(x_{1}, \frac{x_{1}}{z}, \frac{x_{2}}{y} ; P_{s}\right) \ll_{s, B} \frac{x_{2}-x_{1}}{x_{2} \log x_{2}} H\left(x_{2}, \frac{x_{1}}{z}, \frac{x_{2}}{y}\right) & \asymp \frac{x_{2}-x_{1}}{x \log x} H\left(x, \frac{x}{z}, \frac{x}{y}\right) \\
& \asymp \frac{x_{2}-x_{1}}{x \log x} H(x, y, z) .
\end{aligned}
$$

Inserting the above inqequality into (4.25) and summing over $x_{1}, x_{2}$ completes the proof of the desired upper bound. The corresponding lower bound is obtained in a similar fashion starting from

$$
H\left(x, y, z ; P_{s}\right) \geq \sum_{x_{1}, x_{2}}\left\{H\left(x_{2}, \frac{x_{2}}{z}, \frac{x_{1}}{y} ; P_{s}\right)-H\left(x_{1}, \frac{x_{2}}{z}, \frac{x_{1}}{y} ; P_{s}\right)\right\}
$$

and using Theorem 1.6 in place of Theorem 1.3. 
We conclude this section with the proof of Theorem 1.8 .

Proof of Theorem 1.8. Let $2 \leq y \leq z \leq x$. Let $P=\prod_{y<p \leq z} p$ be the product of all prime numbers in $(y, z]$. Then

$$
0 \leq \pi(x-s)-H\left(x, y, z ; P_{s}\right) \leq|\{p \leq x-s:(p+s, P)=1\}| .
$$

Lemma 2.5 implies that the right hand side of (4.26) is

$$
\ll_{s} \frac{x}{\log x} \frac{\log y}{\log z},
$$

which combined with the Prime Number Theorem completes the proof.

Acknowledgements. The author would like to thank Kevin Ford for many valuable suggestions.

\section{REFERENCES}

1. W. R. Alford, A. Granville and C. Pomerance, There are infinitely many Carmichael numbers, Ann. of Math. (2) 139 (1994), no. 3, 703-722.

2. A. S. Besicovitch, On the density of certain sequences of integers, Math. Ann. 110 (1934), 336-341.

3. E. Bombieri, J. B. Friedlander and H. Iwaniec, Primes in arithmetic progressions to large moduli, Acta Math. 156 (1986), no. 3-4, 203-251.

4. H. Davenport, Multiplicative Number Theory, third. ed., Graduate Texts in Mathematics, vol. 74, Springer-Verlag, New York, 2000, Revised and with a preface by Hugh L. Montgomery.

5. P. T. D. A. Elliott and H. Halberstam, A conjecture in prime number theory, Symp. Math., 4 (INDAM Rome, 1968/69), 59-72.

6. P. Erdős, Notes on the sequences of integers no one of which is divisible by any other, J. London Math. Soc. 10 (1935), 126-128.

7. P. Erdős, A generalization of a theorem of Besicovitch, J. London Math. Soc. 11 (1936), 92-98.

8. P. Erdős, Some remarks on number theory, Riveon Lematematika 9 (1955), 45-48, (Hebrew. English summary).

9. P. Erdős, An asymptotic inequality in the theory of numbers, Vestnik Leningrad Univ. 15 (1960), 41-49 (Russian).

10. G. B. Folland, Real Analysis. Modern Techniques and their Applications, second ed., Pure and Applied Mathematics (New York), A Wiley-Interscience Publication, John Wiley and Sons, Inc., New York, 1999.

11. K. Ford, The distribution of integers with a divisor in a given interval, Annals of Math. (2) 168 (2008), 367-433.

12. K. Ford, M. R. Khan, I. E. Shparlinski and C. L. Yankov, On the maximal difference between an element and its inverse in residue rings, Proc. Amer. Math. Soc. 133 (2005), no.12, 3463-3468.

13. J. Friedlander and H. Iwaniec, On Bombieri's asymptotic sieve, Ann. Scuola Norm. Sup. Pisa Cl. Sci (4) 5 (1978), 719-756.

14. H. Halberstam and H. -E. Richert, Sieve Methods, Academic Press [A subsidiary of Harcourt Brace Jovanovich, Publishers], London-New York, 1974, London Mathematical Society Monographs, No. 4.

15. R. R. Hall and G. Tenenbaum, Divisors, Cambridge Tracts in Mathematics, vol. 90, Cambridge University Press, Cambridge, 2008.

16. G. Harman, On the number of Carmichael numbers up to $x$, Bull. London Math. Soc. 37 (2005), 641-650.

17. K. H. Indlekofer and N. M. Timofeev, Divisors of shifted primes, Publ. Math. Debrecen 60 (2002), no. 3-4, 307-345.

18. H. Iwaniec, Rosser's sieve, Acta Arith. 36 (1980), 171-202. 
19. H. Iwaniec, A new form of the error term in the linear sieve, Acta Arith. 37 (1980), 307-320.

20. H. L. Montgomery, Zeroes of L-functions, Invent. Math. 8 (1969), 346-354.

21. H. L. Montgomery and R.C. Vaughan, The large sieve, Mathematica 20 (1973), 119-134.

22. M. Nair and G. Tenenbaum, Short sums of certain arithmetic functions, Acta Math. 180 (1998), 119-144.

23. R. Sitaramachandra Rao, On an error term of Landau, Indian J. Pure Appl. Math. 13 (1982), no. 8, $882-885$.

24. G. Tenenbaum, Lois de répartition des diviseurs. II, Acta Arith. 38 (1980/81), 1-36.

25. G. Tenenbaum, Lois de répartition des diviseurs. III, Acta Arith. 39 (1981), 19-31.

26. G. Tenenbaum, Sur la probabilité qu 'un entier posséde un diviseur dans un intervalle donné, Compositio Math. 51 (1984), no. 2, 243-263.

27. A. Walfisz, Weylsche Exponentialsummen in der Neuen Zahlentheorie, Mathematische Forschungsberichte, XV. VEB Deutscher Verlag der Wissenschaften, Berlin 1963, 231 pp.

Department of Mathematics, University of Illinois at Urbana-Champaign, 1409 West Green Street, Urbana, IL 61801, U.S.A.

E-mail address: dkoukou2@math.uiuc.edu 